\title{
A high-cycle accumulation model for clay and its application to monopile foundations
}

\author{
Patrick Staubach $^{1,2}$ (D) Jan Machaček ${ }^{1,3}$ (D) $\cdot$ Merita Tafili $^{1,4}$ (D) $\cdot$ Torsten Wichtmann $^{1}$ (D)
}

Received: 9 April 2021 / Accepted: 19 December 2021 / Published online: 18 January 2022

(C) The Author(s) 2022

\begin{abstract}
A high-cycle accumulation (HCA) model predicting the accumulation of permanent strain or excess pore water pressure in clay under a large number of load cycles is presented. Data from an extensive laboratory testing program on kaolin under undrained cyclic loading has been analysed for that purpose. The influence of strain amplitude, void ratio, stress ratio, overconsolidation ratio and loading frequency on the accumulation rates is considered in the constitutive equations of the HCA model. The proposed model is validated first by the simulation of element tests. Subsequently, its application to offshore wind turbine foundations under long-term lateral cyclic loading is presented by the back-analysis of a centrifuge test on a monopile in soft clay. The results are in good accordance with the measurements in terms of pile displacement and bending moment versus number of applied cycles. It is concluded that the proposed model is feasible to describe the longterm behaviour of clay subjected to high-cyclic loading.
\end{abstract}

Keywords AVISA model · Clay · High-cycle accumulation model · High-cyclic loading · Offshore wind turbine foundations

\begin{tabular}{|c|c|c|c|}
\hline & $\mathrm{E}$ & Fourth-order stiffness tensor \\
\hline$N$ & t of symbols (ordered by appearance) & $\dot{\boldsymbol{\varepsilon}}$ & Strain rate \\
\hline & Strain amplitude & $\dot{\boldsymbol{\varepsilon}}^{\mathrm{acc}}$ & Strain accumulation rate \\
\hline$\eta$ & Stress ratio & $\dot{\boldsymbol{\varepsilon}}^{\mathrm{pl}}$ & Plastic strain rate \\
\hline$p$ & Mean effective stress & $t_{\text {cycle }}$ & Period of a single cycle \\
\hline$q$ & Deviatoric stress & m & Direction of accumulation \\
\hline \multirow{2}{*}{$\begin{array}{l}\Delta \varepsilon \\
D\end{array}$} & Strain increment & $f_{\mathrm{ampl}}$ & Function of the HCA model considering the strain \\
\hline & Pile diameters & & amplitude \\
\hline \multirow{2}{*}{$\begin{array}{l}\varepsilon^{\mathrm{av}} \\
t\end{array}$} & Average strain & $\dot{f_{N}}$ & Function of the HCA model considering the cyclic \\
\hline & Physical time & & history \\
\hline$\varepsilon^{\max }$ & Maximum strain during a cycle in 1D & $f_{e}$ & Function of the HCA model considering the void \\
\hline$\varepsilon^{\min }$ & Minimum strain during a cycle in 1D & & ratio \\
\hline \multirow{3}{*}{$\begin{array}{l}\sigma \\
\dot{\sigma}\end{array}$} & Effective stress & $f_{Y}$ & Function of the HCA model considering the stress \\
\hline & Effective stress rate & & obliquity \\
\hline & & $f_{\pi}$ & Function of the HCA model considering the \\
\hline \multirow{2}{*}{$\triangle \mathrm{P}$} & ck Staubach & & changes of the cyclic loading direction \\
\hline & k.staubach@rub.de & $g^{A}$ & Cyclic history state variable of the HCA model \\
\hline \multirow{3}{*}{1} & & $e$ & Void ratio \\
\hline & of Soil Mechanics, Foundation Engineering and & $p^{\mathrm{av}}$ & Average mean effective stress \\
\hline & um, Germany & $\eta^{\text {av }}$ & Average stress ratio \\
\hline 2 & $\begin{array}{l}\text { of Geotechnics, Bauhaus-Universität Weimar, Weimar, } \\
\text { lany }\end{array}$ & $\bar{Y}^{\mathrm{av}}$ & $\begin{array}{l}\text { Normalized (Matsuoka \& Nakai) average stress } \\
\text { ratio }\end{array}$ \\
\hline \multirow[t]{2}{*}{3} & ute of Geotechnics, Technische Universität Darmstadt, & $w_{L}$ & Liquid limit \\
\hline & istadt, Germany & $w_{P}$ & Plastic limit \\
\hline 4 & ng. Orth GmbH, Ettlingen, Germany & $I_{v}$ & Viscosity index \\
\hline
\end{tabular}




\begin{tabular}{|c|c|c|c|}
\hline$w$ & Water content & $\boldsymbol{u}$ & Displacement of the solid phase \\
\hline$d$ & Diameter of soil sample in triaxial test & $p^{w}$ & Pore water pressure \\
\hline$h$ & Height of soil sample in triaxial test & $\boldsymbol{u}^{w}$ & Displacement of the water phase \\
\hline$q^{\mathrm{ampl}}$ & Deviatoric stress amplitude in cyclic tests & $K^{\text {Perm }}$ & Permeability \\
\hline$\dot{s}$ & Displacement rate & $\eta^{w}$ & Dynamic viscosity of water \\
\hline OCR & Overconsolidation ratio & $k^{w}$ & Hydraulic conductivity \\
\hline$\dot{u}^{\mathrm{acc}}$ & Rate of pore pressure accumulation & $\gamma^{w}$ & Specific weight of water \\
\hline$u^{\text {acc }}$ & Accumulated pore pressure & $v_{h}$ & Parameter of the AVISA model \\
\hline$\sigma^{\mathrm{av}}$ & Average effective stress & $\alpha$ & Parameter of the AVISA model \\
\hline \multirow{2}{*}{$f_{\mathrm{OCR}}$} & Function of the HCA model considering the & $M_{c}$ & Parameter of the AVISA model \\
\hline & overconsolidation ratio & $e_{i 0}$ & Parameter of the AVISA model \\
\hline \multirow[t]{2}{*}{$f_{f}$} & Function of the HCA model considering the & $f_{b 0}$ & Parameter of the AVISA model \\
\hline & loading frequency & $R$ & Parameter of the AVISA model \\
\hline \multirow[t]{2}{*}{$f_{\eta}$} & Function of the HCA model considering the & $m_{R}$ & Parameter of the AVISA model \\
\hline & average stress ratio & $d$ & Parameter of the AVISA model \\
\hline$C_{\mathrm{ampl}}$ & Parameter of the HCA model in function $f_{\mathrm{ampl}}$ & $\beta_{0}$ & Parameter of the AVISA model \\
\hline$K$ & Bulk modulus & $\chi_{0}$ & Parameter of the AVISA model \\
\hline \multirow[t]{2}{*}{$m_{v}$} & Volumetric portion of the direction & $\chi_{\max }$ & Parameter of the AVISA model \\
\hline & accumulation & $C_{a}$ & Parameter of the AVISA model \\
\hline$\varepsilon_{\text {ref }}^{\text {ampl }}$ & Reference strain amplitude & $H^{\mathrm{av}}$ & Average horizontal load \\
\hline$C_{N 1}$ & Parameter of the HCA model in function $f_{N}$ & $H^{\mathrm{ampl}}$ & Amplitude of horizontal load \\
\hline$C_{N 2}$ & Parameter of the HCA model in function $f_{N}$ & $\Delta p^{w}$ & Excess pore water pressure \\
\hline$C_{N 3}$ & Parameter of the HCA model in function $f_{N}$ & $p_{0}$ & Initial mean effective stress \\
\hline \multirow{2}{*}{$\dot{f}_{N}^{A}$} & N-dependent function of the HCA model consid- & $\Delta \varepsilon^{p l}$ & Increment of plastic strain \\
\hline & ering the cyclic history & $\Delta \phi$ & Increment of plastic potential \\
\hline$\dot{f_{N}^{B}}$ & $\begin{array}{l}\text { Constant function of the HCA model considering } \\
\text { the cyclic history }\end{array}$ & $\vartheta$ & $\begin{array}{l}\text { Scalar factor used for the calculation of the plastic } \\
\text { strain rate }\end{array}$ \\
\hline$\dot{g}^{A}$ & $\begin{array}{l}\text { Rate of the cyclic history state variable of the } \\
\text { HCA model }\end{array}$ & $p_{\text {ref }}$ & $\begin{array}{l}\text { Reference mean effective stress for the AVISA } \\
\text { model }\end{array}$ \\
\hline$p_{\text {atm }}$ & Atmospheric pressure & $\rho^{s}$ & Grain density \\
\hline$e_{\text {ref }}$ & Reference void ratio & $\rho^{w}$ & Water density \\
\hline$e_{\max }$ & Maximum void ratio at loosest possible state & & \\
\hline$C_{e}$ & Parameter of the HCA model in function $f_{e}$ & & \\
\hline$\varphi_{c}$ & Critical friction angle & & \\
\hline$M$ & Critical stress ratio & & \\
\hline
\end{tabular}

$\dot{\varepsilon}_{v}^{\text {acc }} \quad$ Volumetric strain accumulation rate

$\dot{\varepsilon}_{q}^{\text {acc }} \quad$ Deviatoric strain accumulation rate

$\bar{\eta}^{\text {av }} \quad$ Normalized average stress ratio

$C_{\eta} \quad$ Parameter of the HCA model in function $f_{\eta}$

$\theta \quad$ Lode's angle

$p_{e}^{+} \quad$ Equivalent mean effective stress

$e_{e 0} \quad$ Referential void ratio

$p_{e 0} \quad$ Referential mean effective stress

$\lambda \quad$ Compression index

$\left(\boldsymbol{\sigma}^{\mathrm{av}}\right)^{*} \quad$ Deviatoric average effective stress

$F \quad$ Scalar value of yield surface

$\dot{\phi} \quad$ Rate of plastic potential

1 Second-order unit tensor

$\mu \quad$ Scalar shear stiffness

I Fourth-order unit tensor

$\kappa \quad$ Swelling index

$v \quad$ Poisson's ratio

\section{Introduction}

Similar to sand, clay can show an accumulation of permanent strain due to cyclic loading with many repetitions. Such a high-cyclic loading is characterized by comparatively small strain amplitudes (larger, however, than for sand) but a considerable number of load cycles. Highcyclic loading is relevant for many structures, the most prominent and actual example being offshore wind turbine (OWT) foundations which are continuously loaded by wind and water waves during their lifetime. A reliable prediction of the permanent rotation of the OWT foundation caused by this loading in the design stage is important, as excessive tilting endangers its serviceability. In the offshore environment, the loading of the foundation will not be perfectly undrained as it is typically assumed in the 
element tests on clay. The accumulation of (volumetric) strain is thus strongly influenced by the consolidation process occurring simultaneously with the cyclic loading, making the problem at hand even more complex. To take into account these influencing factors and to allow for a reliable estimation of the permanent deformations of OWT foundations subjected to millions of load cycles, the application of numerical methods in combination with special constitutive models can represent a feasible approach. Up to now, only a small number of constitutive models for the prediction of the mechanical response of clay subjected to a large number of load cycles $\left(N \gtrsim 10^{3}-10^{4}\right)$ exist (see e.g. the recently proposed model in [19]). In addition to the enormous and unacceptable time required to calculate this large number of load cycles, conventional constitutive models also fail due to a strong overestimation of the residual strains and a considerable numerical error at large numbers of load cycles, the first resulting from the constitutive equations and the latter owed to the large number of calculated increments [55]. In order to account for millions of load cycles, as faced in the offshore environment, special constitutive models such as the so-called high-cycle accumulation (HCA) models can be applied. These models are formulated in general stressstrain rate form, but do not predict the soil response during individual cycles. Only the average trend of the soil state during the cyclic loading is calculated. Opposite to existing methods used to predict the long-term behaviour of piles subjected to cyclic loading, such as $p-y$ curves or strain wedge models, the application of the HCA models is not restricted to a certain boundary value problem. HCA models are suitable to study arbitrary problems with highcyclic loading. For the prediction of the high-cyclic response of sand, the HCA model by Niemunis et al. [55] is well established [39, 44, 57, 63, 64, 68, 79, 83]. A corresponding model for clay is missing up to now. The development of a HCA model for unstructured clay is thus the aim of the study presented in this paper.

The data of an extensive laboratory testing program on kaolin under undrained cyclic loading are analysed for this purpose. The development of the constitutive equations is presented and the model is validated by the back-analysis of the element tests. To show the applicability and robustness of the proposed model and to extend its validation to a larger scale, a monopile foundation for OWTs under cyclic lateral loading is studied in the framework of a back-analysis of a centrifuge model test.

\section{Literature review on clay behaviour under cyclic loading}

The literature review in this section concentrates on three aspects being of relevance for the development and validation of the HCA model: 1) the behaviour of clay under cyclic loading in laboratory tests, which has to be reflected by the HCA model equations, 2) available conventional constitutive models for clay under cyclic loading which are needed for the low-cycle phases of the HCA simulations as described in Section 3, and 3) model tests with cyclic lateral loading on piles in clay, from which a specific centrifuge test is selected for the validation of the HCA model by means of a back analysis presented in Section 5 .

\subsection{Laboratory tests with cyclic loading on clay}

Laboratory tests with cyclic loading on clay are usually performed under undrained conditions. The cyclic loading can lead to a build-up of excess pore water pressure (i.e. effective stress relaxation) and an accumulation of shear strain under those circumstances. Cumulative effects (i.e. build-up of excess pore water pressure and accumulation of shear strain) in clay start if the strain amplitude exceeds a certain threshold value of the strain amplitude $\varepsilon^{\text {ampl }}$. This threshold strain amplitude increases with increasing plasticity of the test material $[32,33,36,76]$. The intensity of effective stress relaxation or strain accumulation increases with the stress or strain amplitude $[1,3,7,12,13,17$, $21,28-30,32-35,51,52,71,76,87,90]$. In undrained cyclic triaxial tests with isotropic average stresses or a relatively small stress anisotropy, clay samples usually fail due to the development of large axial strain amplitudes, exceeding a pre-defined failure criterion (e.g. $10 \%$ ). In tests with larger stress ratios $|\eta=q / p|$ an excessive accumulation of permanent strain usually leads to failure $[1,3,35,80]$.

Some experimental studies reported that a multidimensional cyclic loading (i.e. at least two stress components oscillate out-of-phase) causes larger cumulative effects than one-dimensional cycles (i.e. only one stress component oscillates or several stress components vary in-phase) $[18,48,70,84]$. At same stress amplitudes, the cumulative rates are lower in overconsolidated clays than in normally consolidated clays. If the stress amplitude is referred to the undrained shear strength $s_{u}$ from monotonic tests, however, the conclusions regarding the tendency are not unique in the literature $[4,21,30,35,90]$. Most laboratory studies 
performed in the framework of research projects were conducted on reconstituted samples, often prepared by reconsolidation out of a slurry. Based on a comparison of undisturbed and remoulded samples of several marine clays in undrained cyclic triaxial tests, Hyodo et al. [35] concluded that data of normally consolidated reconstituted samples define an intrinsic cyclic strength. This intrinsic cyclic strength is increased by aging effects, cementation and overconsolidation. There is a consensus in the literature that an increase in the loading frequency leads to a decrease in the accumulation rates $[5,14,30,49,51$, 87, 90]. The influence of frequency grows with the plasticity of the clay [1]. Generally, the rates of pore water pressure build-up and shear strain accumulation decrease with increasing plasticity $[1,13,27,35,37]$.

\subsection{Conventional constitutive models for clay under low-cycle loading}

The cyclic behaviour of fine-grained soils depends not only on the material state, such as the effective stress or the density, but also on the strain amplitude and the strain rate. The clay behaves elastically only under very small strain amplitudes of about $\varepsilon^{\mathrm{ampl}}<10^{-4}$. Under medium strain amplitudes $10^{-4}<\varepsilon^{\mathrm{ampl}}<10^{-2}$ the stiffness increase at load reversals is accompanied by a reduction in the plastic strain rate $[15,80]$. With increasing distance to the reversal point, the stiffness decreases again, as observed on a typical stiffness degradation curve. Finally, when the soil is subjected to shearing with large strains $\| \Delta \varepsilon||>10^{-2}$ it tends asymptotically to the critical state corresponding to failure under monotonic loading and resulting in cyclic mobility under cyclic loading. These are some relevant aspects of clay behaviour which are expected to be considered by competent constitutive models.

A single model capturing all these effects is rare to find if not impossible, and therefore, researchers recommend the usage of an "appropriate" model for each particular problem. For example, one could choose an elastoplastic model (e.g. Modified Cam Clay (MCC) model [11]), a hypoplastic model $[46,74]$ or a barodesy model [50] for the simulation of a bearing capacity problem of a shallow foundation on clay. These models are only able to simulate monotonic loading for non-viscous clays, and as in detail discussed in [72], they would not capture the strain rate dependency typical for plastic clays (as is the case with soft marine clays). If the clay shows a high plasticity, a ratedependent model could be useful $[10,86]$. These models are, however, again restricted to monotonic loading conditions.

For cyclic loading with low to medium number of cycles, the constitutive model should certainly consider small-strain and memory effects. For that purpose, hypoplastic models can be extended by the intergranular strain concept [56] or the intergranular strain anisotropy (ISA-plasticity) [24]. For example, the visco-hypoplastic model of 2003 [53] includes the intergranular strain tensor, while the later version of 2009 [54] considers induced anisotropy, but no small-strain effects. Alternatively, the SANICLAY model [61] can be applied. A substantial drawback of most of these models is the incapability to adequately describe the soil behaviour under different loading amplitudes with the same set of material parameters. Intending to embrace a wide range of strain amplitudes with a single constitutive equation the ISA-plasticity for sand $[24,25]$ was reformulated by Tafili \& Triantafyllidis [73] to account for the specific behaviour of clays. This proposed anisotropic visco-ISA (AVISA) model incorporates the rate- and time-dependency of clays as well as inherent anisotropy. In 2020, the ISA-plasticity model was coupled with the hypoplastic model for finegrained soils developed by Mašín [26]. This model also accounts for the inherent anisotropy of fine-grained soils, but neglects rate- and time-dependent effects. In the same year, barodesy has been coupled with the intergranular strain concept in [9], however, without introducing the inherent anisotropy or the time-dependency into the model.

\subsection{Piles in clay subjected to cyclic loading}

Due to cyclic lateral loading, a reduction in stiffness of the fine-grained soil surrounding a pile with increasing number of cycles occurs, which is mainly a result of a build-up of excess pore water pressure. This has for instance been observed in field tests with up to one thousand lateral load cycles on piles (diameters $D=0.273 \mathrm{~m}$ and $D=1.22 \mathrm{~m}$ ) in stiff overconsolidated clay [22]. Similar results were obtained in recent field tests on monopile foundations with a diameter of $2.2 \mathrm{~m}$ in soft clay reported in [91] as well as in the pioneer works of Matlock [47] (in soft clay) and Reese et al. [60] (in heavily overconsolidated clay). In [77] a strong degradation of the lateral stiffness of the pile ( $D=0.8 \mathrm{~m}$, founded in soft clay) was observed during the cyclic loading as soon as the magnitude of the load was larger than a certain threshold level. The largest degradation occurred during the first five cycles followed by a stagnation of degradation. In case of cyclic loading with even higher load magnitude, the rate of reduction in pile stiffness increased with increasing number of applied cycles without showing the aforementioned stagnation. Similar observations were made for piles with a diameter of $D=0.153 \mathrm{~m}$ in overconsolidated clay reported in [89]. Recently, Yang et al. [85] have performed centrifuge tests on monopile foundations ( $D=5.9 \mathrm{~m}$ in prototype scale) in soft kaolin clay subjected to lateral cyclic loading with 
hundreds of load cycles. These tests are considered for the back-analysis presented in Sect. 5 applying the HCA model for clay proposed hereinafter. In accordance with the observations documented in [58, 59], the cyclic loading lead to an increase in the magnitude of the bending moment and a decrease in the pile stiffness. Small scale model tests on piles $(D=50 \mathrm{~mm})$ in marine clay reported in [41] with several thousand lateral load cycles showed an increasing permanent deflection of the pile for cyclic amplitudes in the range of $30 \%$ to $60 \%$ of the ultimate lateral resistance. Alternate cyclic loading with phases of reconsolidation between the cyclic events in centrifuge tests on monopiles $(D=4 \mathrm{~m}$ and $D=6 \mathrm{~m}$ in prototype scale) in soft clay revealed a partial recovery of the degraded lateral pile stiffness due to the resting period [40]. However, within each cyclic load package, the cumulative displacement of the pile increased with number of applied load cycles. Recently performed field tests on piles of different size $(D=0.273 \mathrm{~m}, D=0.762 \mathrm{~m}$ and $D=2 \mathrm{~m})$ installed in an overconsolidated clay employing different rates of lateral monotonic loading showed that the piles exhibited a much higher resistance for higher load rates [16]. In addition, the formation of a gap between soil and pile was reported for all tests, which is believed to contribute to the reduction in the stiffness of the pile system when subjected to cyclic lateral loading.

A reduction in soil reaction force with ongoing cyclic lateral loading is incorporated in a simplified manner in the cyclic $p-y$ curves of the American Petroleum Institute (API) [6] which are based on field tests [47]. Since the API cyclic $p-y$ curves do not allow for a consideration of the number of applied load cycles or the load characteristics (e.g. the ratio of average and cyclic load), many modifications have been proposed over the span of the last decades. Such improved cyclic $p-y$ curves have for instant recently been presented in [88], where the reduction in the soil reaction with ongoing cyclic loading has been incorporated using cyclic strain contour diagrams obtained from direct simple shear tests (see [2]). An extension of the $p-y$ curves considering rate effects has been presented in [8].

Simulations of centrifuge tests on monopiles subjected to lateral cyclic loading in soft clay using a hypoplastic model with intergranular strain extension have been presented in $[23,31]$. However, due to the nature of conventional constitutive models, only a limited number of load cycles can be taken into account by such an approach. In order to consider millions of load cycles, special constitutive models such as the HCA model are necessary.

\section{General concept of a HCA model and the HCA model for sand}

In the framework of a HCA model the strain resulting from cyclic loading is split in its oscillating part $\varepsilon^{\text {ampl }}$ and its average part $\varepsilon^{\text {av }}$. The constitutive model works merely in terms of average values and calculates $\varepsilon^{\text {av }}$ only. Thus, the time $t$ can be replaced by the number of cycles $N$. For cyclic loading with a large number of cycles this is advantageous since it is not necessary to consider individual cycles during the simulation. This reduces not only the computational effort considerably but also avoids an accumulation of numerical errors within each increment, considering that millions of increments are necessary for the simulation of a large number of cycles.

Two numerical strategies are combined in a simulation with the HCA model. They are termed the low-cycle (conventional, implicit) and the high-cycle (N-type, explicit) mode of calculation. The low-cycle mode uses a conventional constitutive model. Monotonic loading phases or a few complete cycles are calculated in this mode. The low cycle-mode is necessary to determine the field of the scalar strain amplitude $\varepsilon^{\text {ampl }}$, which is an important input parameter of the HCA model. The strain amplitude is determined in each integration point of each finite element from the tensorial strain path recorded during an individual load cycle calculated in the low-cycle mode. As depicted in Fig. 1, this can either be the second cycle or a so-called

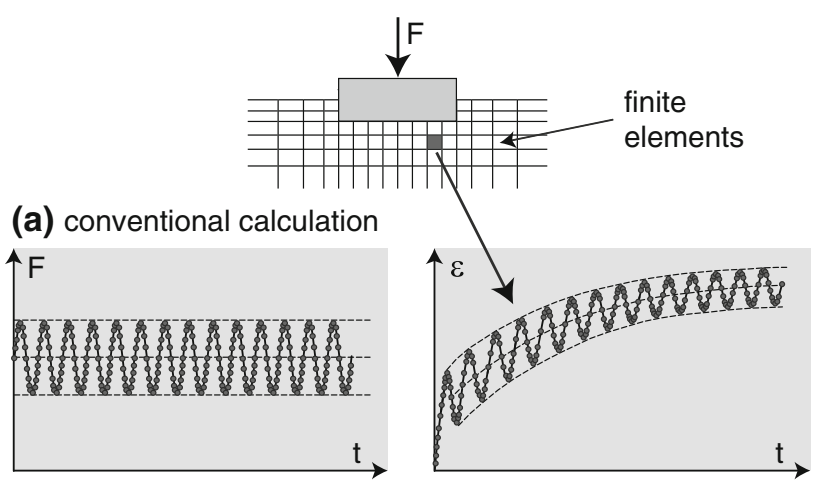

(b) calculation with a high-cycle accumulation (HCA) model
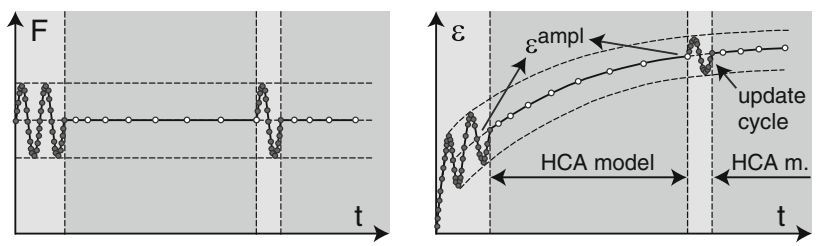

Fig. 1 FE simulations of a shallow foundation under cyclic loading: a) Pure low-cycle (conventional) versus b) combined low-cycle and high-cycle mode. The strain amplitude $\varepsilon^{\text {ampl }}$ is determined from the second loading cycle. It can be updated after a given number of cycles by interrupting the high-cycle phase by additional (update) cycles in the low-cycle mode 
update cycle. The first cycle should not be used for the calculation of the strain amplitude, because the first quarter of the first cycle represents an initial loading, while all further cycles represent un- and reloading. Based on the recorded tensorial strain path (six independent components of the strain tensor for $3 \mathrm{D}$ cases), the strain amplitude is defined as the Euclidean norm of six spans obtained by consecutive projection on (hyper-) planes (for details see the work presenting the HCA model for sand [55]). For one-dimensional strain paths this definition of a multidimensional strain amplitude simplifies to the classical definition $\varepsilon^{\mathrm{ampl}}=\left(\varepsilon^{\mathrm{max}}-\varepsilon^{\mathrm{min}}\right) / 2$. This amplitude definition is also adopted for the HCA clay model proposed in this work. During the high-cycle phases the strain amplitude is assumed constant. The field of the strain amplitude can be updated using an update cycle, which is simulated in the low-cycle mode again.

In the high-cycle mode the HCA model is used to predict the development of the average values of strain and effective stress. The basic equation of the HCA model for sand reads

$\dot{\boldsymbol{\sigma}}=\mathrm{E}:\left(\dot{\boldsymbol{\varepsilon}}-\dot{\boldsymbol{\varepsilon}}^{\mathrm{acc}}-\dot{\boldsymbol{\varepsilon}}^{\mathrm{pl}}\right)$

with the (objective) stress rate $\dot{\sigma}$ of the effective Cauchy stress $\boldsymbol{\sigma}$ (compression positive), the strain rate $\dot{\boldsymbol{\varepsilon}}$ (compression positive), the accumulation rate $\dot{\boldsymbol{\varepsilon}}^{\text {acc }}$, a plastic strain rate $\dot{\boldsymbol{\varepsilon}}^{\mathrm{pl}}$ (necessary only for stress paths touching the yield surface) and the pressure-dependent elastic stiffness E. In the framework of HCA models the dot over a symbol denotes a derivative with respect to the number of cycles $N$, i.e. $\sqcup \dot{ }=\partial \sqcup / \partial N$. Note that when integrating Eq. (1) numerically in a finite-element (FE) analysis it has to be integrated over $\Delta N=\Delta t / t_{\text {cycle }}$ where $t_{\text {cycle }}$ is the duration of a single cycle (period) and $\Delta t$ is the time increment applied by the FE program. In other words, although the constitutive equations of the HCA model are integrated with respect to $N$, the simulation is performed in time domain. Therefore, the modelling of transient processes is possible as with regular constitutive models. Depending on the boundary conditions, Eq. (1) predicts a change of stress $(\dot{\boldsymbol{\sigma}} \neq \mathbf{0})$ and / or an accumulation of strain $(\dot{\boldsymbol{\varepsilon}} \neq \mathbf{0})$. For the HCA model for sand, the rate of accumulated strain in Eq. (1) is calculated from:

$\dot{\boldsymbol{\varepsilon}}^{\mathrm{acc}}=\dot{\varepsilon}^{\mathrm{acc}} \mathbf{m}=f_{\mathrm{ampl}} \dot{f_{N}} f_{e} f_{p} f_{Y} \mathbf{m}$

The factors $f_{\sqcup}$ of the intensity of accumulation $\dot{\varepsilon}^{\text {acc }}$ take into account the influence of the strain amplitude $\varepsilon^{\text {ampl }}$ (function $\left.f_{\text {ampl }}\right)$, the cyclic preloading $\left(\dot{f_{N}}\right.$, using the preloading variable $g^{A}$ which weights the number $N$ of applied cycles with the strain amplitude $\varepsilon^{\text {ampl }}$ of these cycles), the void ratio $e\left(f_{e}\right)$, the average mean effective stress $p^{\text {av }}\left(f_{p}\right)$ and the normalized average stress ratio $\bar{Y}^{\mathrm{av}}\left(f_{Y}, \bar{Y}^{\mathrm{av}}=0\right.$ at isotropic stresses, $\bar{Y}^{\mathrm{av}}=1$ at critical stress ratio). Note that the factor $f_{\pi}$ describing the influence of changes of the cyclic loading direction used in previous publications on the model has been omitted here, because a recent experimental study proved it to be of minor importance [82]. $\mathbf{m}$ is the direction of accumulation (unit tensor), which determines the ratio of the rates of volumetric and deviatoric strain accumulation.

\section{HCA model for clay}

Equation (1) used in the HCA model for sand is adopted for the clay model as well. The stiffness E, the intensity of accumulation $\dot{\varepsilon}^{\text {acc }}$, the plastic strain rate $\dot{\boldsymbol{\varepsilon}}^{\mathrm{pl}}$ and the direction of accumulation $\mathbf{m}$ have to be adjusted for the clay model, however. In the following, the development of suitable functions for $\dot{\varepsilon}^{\text {acc }}$ (Sect. 4.1) is presented. The investigation of $\mathbf{m}$ is presented in Sect. 4.2, while $\mathrm{E}$ and $\dot{\boldsymbol{\varepsilon}}^{\mathrm{pl}}$ are discussed in Sect. 4.3.

In order to study the influencing factors on the intensity of accumulation, an extensive laboratory testing program with undrained cyclic triaxial tests has been conducted on reconstituted kaolin clay (liquid limit $w_{L}=47.2 \%$, plastic limit $w_{P}=12.2 \%$ and viscosity index $I_{v}=1.5 \%$ ). All samples were consolidated out of a slurry (water content of $w / w_{L}=2.5$ ). Dry kaolin powder was mixed with demineralized water under vacuum for at least 12 hours. The slurry was pre-consolidated in a consolidation apparatus. The axial loading during this pre-consolidation was applied pneumatically. The pre-consolidated samples had a diameter of $d=130 \mathrm{~mm}$ and a height of $h \approx 130 \mathrm{~mm}$. Afterwards, triaxial samples $(d=h=50 \mathrm{~mm})$ were cut out of the centre of the preconsolidated sample. In several test series the overconsolidation ratio OCR, the initial mean effective stress $p_{0}$, the initial stress ratio $\eta_{0}=q_{0} / p_{0}$, the stress amplitude $q^{\text {ampl }}$ and the displacement rate $\dot{s}$ have been varied. If not varied, the cyclic loading was applied with a constant displacement rate $\dot{s}=0.1 \mathrm{~mm} / \mathrm{min}$. A more elaborated description of the triaxial tests as well as a detailed examination of the results can be found in $[78,80]$. In the following, only those parts of the results required for the development of the constitutive equations are presented.

The interval between the cycles 1 and 5 has been used for the evaluation of the rate of pore pressure accumulation $\dot{u}^{\text {acc }}$, i.e. $\dot{u}^{\text {acc }}=\left[u^{\text {acc }}(N=5)-u^{\text {acc }}(N=1)\right] / 4$. Since the average stress and the strain amplitudes continuously change with the number of load cycles $N$ during the undrained cyclic loading, the analysis is restricted to the early stage of the tests. Compared to sand, where data from drained cyclic tests with almost constant values of $\boldsymbol{\sigma}^{\text {av }}$ and 
$\varepsilon^{\text {ampl }}$ are usually available, the calibration of the HCA model for clay based on undrained cyclic test data is thus more challenging. It should be noted that the data of the first cycles is used to derive suitable equations and to undertake a first manual calibration of the material constants. This applies to all factors $f_{\sqcup}$ discussed in this and the following subsection. A further inspection of the equations and a fine-tuning of the material constants is done based on the element test simulations presented in Section 4.5.

\subsection{Intensity of accumulation}

Compared to sand, additional functions to take into account the overconsolidation ratio OCR $\left(f_{\mathrm{OCR}}\right)$ and the loading frequency $\left(f_{f}\right)$ have to be added to the intensity of accumulation for the clay model. On the other hand, one of the functions $f_{e}$ and $f_{p}$ incorporated in the HCA model for sand can be omitted since in normally consolidated soils void ratio and pressure are directly interrelated to each other via the normal compression curve $e(p)$. It has been decided to keep $f_{e}$. The following multiplicative approach is used for the intensity of strain accumulation:

(a)

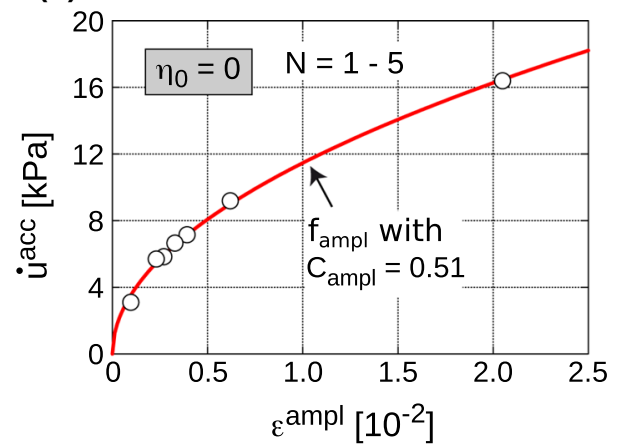

(b)

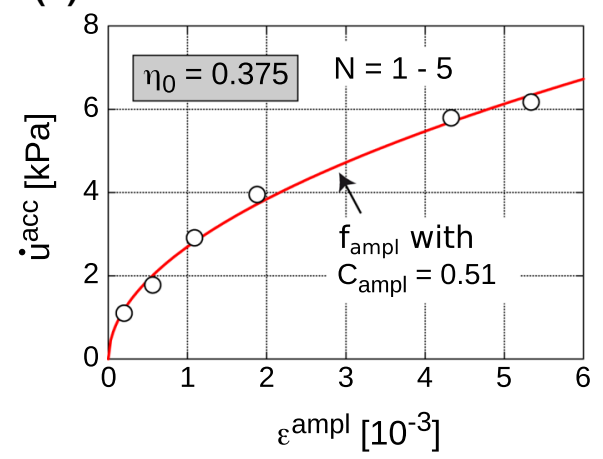

Fig. 2 Calibration of parameter $C_{\text {ampl }}$ of function $f_{\text {ampl }}$ based on the undrained cyclic tests with different amplitudes performed on samples consolidated either a) isotropically $\left(p_{0}=200 \mathrm{kPa}, \eta_{0}=0\right)$ or b) anisotropically $\left(p_{0}=200 \mathrm{kPa}, \eta_{0}=0.375\right)$ $\dot{\varepsilon}^{\mathrm{acc}}=f_{\mathrm{ampl}} \dot{f_{N}} f_{e} f_{\eta} f_{O C R} f_{f}$

In addition to the aforementioned modifications, the factor $f_{Y}$ of the model for sand has been replaced by $f_{\eta}$ in Eq. (3). As has been shown in [78], the definition of this factor in terms of normalized average stress ratio $\bar{\eta}^{\text {av }}$ is more convenient than using $\bar{Y}^{\mathrm{av}}$ employed for the HCA sand model.

\subsubsection{Influence of the strain amplitude $\left(f_{\text {ampl }}\right)$}

The influence of the strain amplitude on the intensity of accumulation and the function $f_{\text {ampl }}$ describing this influence is inspected in Fig. 2, where the rates of pore pressure accumulation $\dot{u}^{\text {acc }}$ measured during the initial phase of the triaxial tests are shown. Two different test series with different initial stress ratios $\eta_{0}=0$ and $\eta_{0}=0.375$ are considered. The initial mean effective stress was $p_{0}=200$ $\mathrm{kPa}$ in both test series, while the stress amplitudes lay in the ranges $30 \mathrm{kPa} \leq q^{\mathrm{ampl}} \leq 70 \mathrm{kPa}\left(\eta_{0}=0\right)$ or $15 \mathrm{kPa}$ $\leq q^{\mathrm{ampl}} \leq 40 \mathrm{kPa}\left(\eta_{0}=0.375\right)$, respectively.

The rate of pore pressure accumulation $\dot{u}^{\text {acc }}$ and the intensity of strain accumulation $\dot{\varepsilon}^{\text {acc }}$ are proportional to each other $\left(\dot{u}^{\mathrm{acc}}=K \dot{\varepsilon}^{\mathrm{acc}} m_{v}\right)$. Since all tests within a series have been started at the same isotropic stress $\left(p_{0}=200 \mathrm{kPa}\right.$, $\eta_{0}=0$ or 0.375 ), the bulk modulus $K$ and the volumetric component of the direction of accumulation $m_{v}$ are assumed constant within the first cycles. Therefore, $\dot{u}^{\text {acc }}$ can be analysed instead of $\dot{\varepsilon}^{\text {acc }}$.

Figure 2 shows $\dot{u}^{\text {acc }}$ as a function of the strain amplitude $\varepsilon^{\text {ampl }}$ (mean value over the range $N=1-4$ ) for both the tests with isotropic $\left(\eta_{0}=0\right.$, Fig. $\left.2 \mathrm{a}\right)$ and anisotropic $\left(\eta_{0}=\right.$ 0.375 , Fig. 2b) initial stresses. Compared to sand, where values below $10^{-3}$ are typically encountered, under similar initial and cyclic stress conditions, the strain amplitudes are much larger for the kaolin clay. Therefore, it is meaningful to use a larger reference strain amplitude $\varepsilon_{\text {ref }}^{\text {ampl }}=10^{-3}$ in the HCA model for clay than in the sand model, where $\varepsilon_{\text {ref }}^{\text {ampl }}=10^{-4}$ has been used. The increase in $u^{\text {acc }}$ with $\varepsilon^{\text {ampl }}$ almost follows a square root function. If the data are fitted by

$\dot{u}^{\mathrm{acc}} \sim f_{\mathrm{ampl}}=\left(\frac{\varepsilon^{\mathrm{ampl}}}{\varepsilon_{\mathrm{ref}}^{\mathrm{ampl}}}\right)^{C_{\mathrm{ampl}}}$

the same exponent $C_{\mathrm{ampl}}=0.51$ is obtained for both test series. The current study indicates that the amplitude dependence is thus much less pronounced than in case of sand for which values in the range of $C_{\mathrm{ampl}}=1.3$ to $C_{\mathrm{ampl}}=2.4$ are usually found [81]. 


\subsubsection{Influence of the cyclic preloading $\left(\dot{f}_{N}\right)$}

Based on tests performed on sand, a suitable function to describe the increase in accumulated strain with increasing number of cycles $N$ was found to be [55]:

$f_{N}=C_{N 1}\left[\ln \left(1+C_{N 2}\right)+C_{N 3} N\right]$

$C_{N 1}, C_{N 2}$ and $C_{N 3}$ are material parameters. The function is adopted for the clay model as well. The required derivative of Eq. (5) with respect to $N$ is given by:

$\dot{f_{N}}=\frac{C_{N 1} C_{N 2}}{1+C_{N 2} N}+C_{N 1} C_{N 3}$

As has been demonstrated in [55], the definition of $\dot{f_{N}}$ in Eq. (6) contradicts the Miner's rule. A certain number (say $10,000)$ of cycles with a strain amplitude tending to zero should lead to zero accumulated strain and have no effect on the accumulation of strain during a subsequent cyclic loading with larger strain amplitudes. However, this is not the case when using the formulation of Eq. (6), since the cyclic loading is solely quantified by the number of cycles, ignoring the corresponding strain amplitude. To resolve this, the intensity of cyclic loading has to be considered in the state variable quantifying cyclic preloading. Therefore, a so-called historiotropic state variable $g^{A}$ was introduced in the original model by Niemunis et al. [55], replacing $\dot{f_{N}}(N)$ by $\dot{f_{N}}\left(g^{A}\right)$, where $g^{A}=f\left(N, f_{\text {ampl }}\right)$ considers both the number of applied cycles and their corresponding intensity by $f_{\text {ampl }}$. The same definition of the function $\dot{f_{N}}$ as in the model for sand is used in the model for clay (for a detailed derivation of the equation the interested reader is referred to [55]). $\dot{f_{N}}$ is split into an $N$-dependent portion $\dot{f}_{N}^{A}$ and a constant portion $f_{N}^{B}$ :

$\dot{f_{N}}=\dot{f}_{N}^{A}+\dot{f}_{N}^{B}$

with:

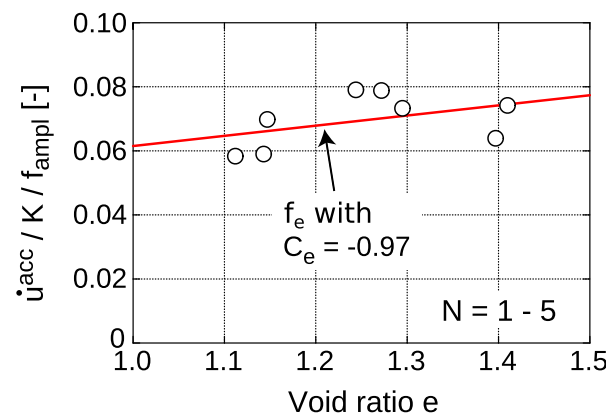

Fig. 3 Calibration of function $f_{e}$ based on the undrained cyclic tests with different initial pressures $p_{0}$
$\dot{f}_{N}^{A}=C_{N 1} C_{N 2} \exp \left[-\frac{g^{A}}{C_{N 1} f_{\mathrm{ampl}}}\right]$

$f_{N}^{B}=C_{N 1} C_{N 3}$

Note that for a non-constant strain amplitude $\varepsilon^{\text {ampl }}(N)$ (and hence non-constant function $f_{\text {ampl }}$ ) the definition of $\dot{f}_{N}^{A}$ becomes [66]:

$f_{N}^{A}=\frac{C_{N 1} C_{N 2}}{\left(1+C_{N 2} N_{0}\right)} \exp \left[\frac{g^{A}\left(N_{0}\right)-g^{A}(N)}{C_{N 1} f_{\text {ampl }}}\right]$

$N_{0}$ is the number of the cycle at which the strain amplitude changed the last time. The rate of the preloading variable $g^{A}$ is defined by:

$\dot{g}^{A}=f_{\text {ampl }} \dot{f}_{N}^{A}$

The parameters $C_{N 1}, C_{N 2}$ and $C_{N 3}$ are determined based on the back-analysis of element tests presented in Section 4.5.

\subsubsection{Influence of the void ratio $\left(f_{e}\right)$}

Instead of $f_{e}$ and $f_{p}$ used in the HCA model for sand, the combined influence of void ratio and pressure is described solely by the void ratio function $f_{e}$ in the model for clay, considering that $e$ and $p$ are directly correlated in normally consolidated clays by the virgin compression curve. Tests with different initial void ratios $e_{0}$ obtained after consolidation at different mean effective initial stresses within the range $50 \mathrm{kPa} \leq p_{0} \leq 300 \mathrm{kPa}$ have been performed for this purpose, with $\eta_{0}=0$ and a constant ratio of stress amplitude and initial pressure $q^{\text {ampl }} / p_{0}=0.2$. Figure 3 displays the results of these tests. The influence of the strain amplitude has been eliminated by dividing $u^{\text {acc }}$ through $f_{\text {ampl }}$. In addition, since the difference in mean effective stress also influences the bulk modulus $K$, the normalized rates $u^{\text {acc }} / f_{\text {ampl }}$ have to be further divided by $K$, which corresponds to a conversion of the rates of pore pressure accumulation $\dot{u}^{\text {acc }}$ into rates of volumetric strain

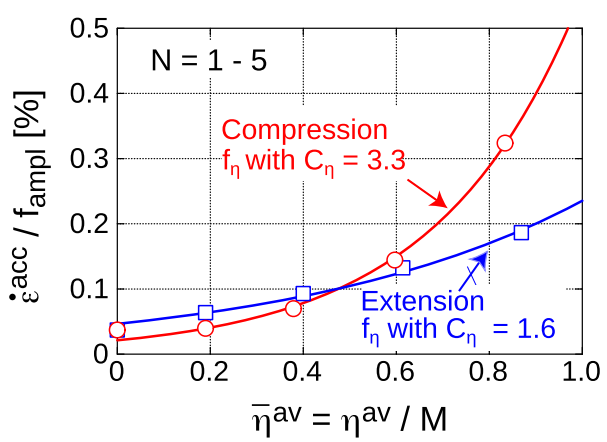

Fig. 4 Calibration of function $f_{\eta}$ by means of the data from undrained cyclic triaxial tests with different initial stress ratios $\eta_{0}$ lying in either the compression or extension regime of the $p-q$ plane 
accumulation $\dot{\varepsilon}_{v}^{\text {acc }}=\dot{u}^{\text {acc }} / K$. The pressure-dependent bulk modulus has been calculated using:

$K=35 p_{\text {atm }}\left(p / p_{\text {atm }}\right)^{1.0}=35 p$

with atmospheric pressure $p_{\text {atm }}$. Equation (12) has been derived from the unloading curve of oedometric compression tests reported in [78], considering that the stress relaxation caused by a cyclic loading represents a kind of unloading as well. The slight increase in $u^{\text {acc }} /\left(K f_{\text {ampl }}\right)$ with $e$ visible in Figure 3 is captured by

$\dot{u}^{\mathrm{acc}} /\left(K f_{\mathrm{ampl}}\right) \sim f_{e}=\frac{\left(C_{e}-e\right)^{2}}{1+e} \frac{1+e_{\mathrm{ref}}}{\left(C_{e}-e_{\mathrm{ref}}\right)^{2}}$

with $C_{e}=-0.97$. The reference void ratio has been arbitrarily chosen as $e_{\text {ref }}=1.25$, lying in the middle of tested void ratios (in the model for sand the void ratio $e_{\max }$ corresponding to the loosest possible state is applied). Obviously, an approximation of the data in Fig. 3 by a linear function would fit as well but Eq. (13) has been chosen in analogy to the HCA model for sand.

\subsubsection{Influence of the stress ratio $\left(f_{\eta}\right)$}

The influence of stress ratio is investigated in Fig. 4, based on the data from tests with different initial stress ratios in the range $-0.5 \leq \eta_{0} \leq 0.625$, i.e. comprising the compression as well as the extension regime. All tests within this series were performed with $p_{0}=200 \mathrm{kPa}$ and $q^{\text {ampl }}=30 \mathrm{kPa}$. The average stress ratio $\eta^{\text {av }}$ used on the horizontal axis in Fig. 4 is the average value of the $\eta$ values during the first five cycles. $M$ denotes the critical stress ratio in compression or extension, respectively, which has been calculated with a critical state friction angle of $\varphi_{c}=$ $19^{\circ}$ (for it's determination see [78]). In general, for a certain average stress ratio $\eta^{\text {av }}$, both an accumulation of pore water pressure and an accumulation of deviatoric strain take place. To analyse a single quantity, the rate of pore pressure accumulation $\dot{u}^{\text {acc }}$ has been converted into a volumetric strain rate $\dot{\varepsilon}_{v}^{\mathrm{acc}}$, before the total strain rate $\dot{\varepsilon}^{\text {acc }}$ is evaluated:

$$
\dot{\varepsilon}^{\mathrm{acc}}=\sqrt{\frac{1}{3}\left(\dot{\varepsilon}_{v}^{\mathrm{acc}}\right)^{2}+\frac{3}{2}\left(\dot{\varepsilon}_{q}^{\mathrm{acc}}\right)^{2}}=\sqrt{\frac{1}{3}\left(\frac{\dot{u}^{\mathrm{acc}}}{K\left(p^{\mathrm{av}}\right)}\right)^{2}+\frac{3}{2}\left(\dot{\varepsilon}_{q}^{\mathrm{acc}}\right)^{2}}
$$

with $p^{\text {av }}$ being the mean effective stress corresponding to $\eta^{\text {av }}$. The intensity of accumulation calculated from Eq. (14) has been plotted versus the normalized stress ratio $\bar{\eta}^{\text {av }}=$ $\eta^{\text {av }} / M$ in Figure 4. The function $f_{\eta}$ describes the influence of the average stress ratio and is chosen as

$f_{\eta}=\exp \left(C_{\eta} \bar{\eta}^{\mathrm{av}}\right)$

with the material constant $C_{\eta}$. The data in Fig. 4 reveal that different parameters $C_{\eta}$ are necessary in order to fit the compression or extension test data. Therefore, an introduction of Lode's angle $\theta$ into $f_{\eta}$ seems recommendable. For such extension, however, the data from additional hollow cylinder tests with cycles applied at different $\theta$ values would be advantageous (i.e. not only the special cases triaxial compression and extension should be tested).

\subsubsection{Influence of the overconsolidation ratio $\left(f_{\mathrm{OCR}}\right)$}

The necessity of a function describing the influence of the overconsolidation ratio OCR is demonstrated in Fig. 5a. Compared to the normally consolidated samples (OCR $=1$, data overtaken from Fig. 3), the pore pressure accumulation rates in the overconsolidated clay (OCR values up to 2.5 have been tested) are much lower, despite having a similar void ratio. The higher OCR, the lower are the $\dot{u}^{\text {acc }} /\left(K f_{\text {ampl }}\right)$ values. The decrease in the intensity of (a)

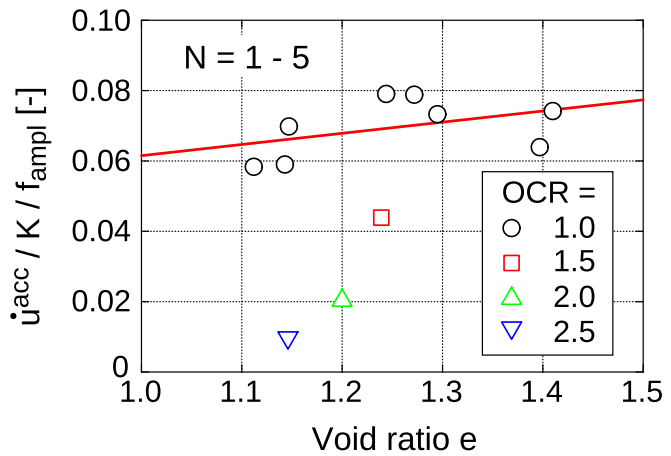

(b)

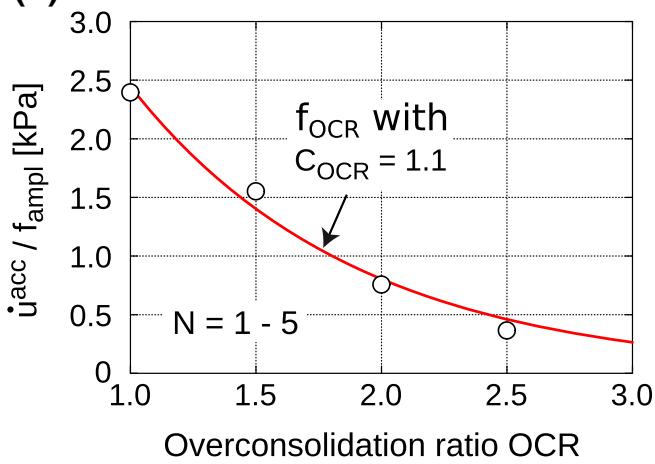

Fig. 5 Pore pressure accumulation rates $\dot{u}^{\text {acc }} /\left(K f_{\text {ampl }}\right)$ or $\dot{u}^{\text {acc }} / f_{\text {ampl }}$, respectively, from tests with different overconsolidation ratios OCR versus a) void ratio $e$ and $\mathrm{b}$ ) OCR. Note that all tests given in plot $\mathrm{b}$ ) have been conducted with an initial mean effective stress $p_{0}=100 \mathrm{kPa}$ for why no normalization with $K$ is required 
accumulation by an overconsolidation is thus much stronger than expected from the change of the void ratio $\Delta e$ caused by the preloading. It is thus impossible to describe the reduction in the cumulative rates with increasing overconsolidation by the void ratio function $f_{e}$ alone. Consequently, an additional function describing the OCR influence is indispensable.

Figure $5 \mathrm{~b}$ shows the normalized pore pressure accumulation rates from the initial phase of the tests with different overconsolidation ratios plotted versus OCR. An exponentially decreasing function has been chosen to describe the relationship:

$\dot{u}^{\mathrm{acc}} / f_{\mathrm{ampl}} \sim f_{\mathrm{OCR}}=\exp \left[-C_{\mathrm{OCR}}(\mathrm{OCR}-1)\right]$

with a material constant $C_{\mathrm{OCR}}=1.1$ derived from the data in Fig. 5b. It has to be considered, however, that OCR $=p_{\text {preload }} / p$ increases during the undrained cyclic test, due to the relaxation of $p$. In Figure $5 \mathrm{~b}$ the initial values $\mathrm{OCR}_{0}=p_{\text {preload }} / p_{0}$ have been plotted on the abscissa, which seems reasonable for the initial phase of the tests, where $p^{\text {av }}$ is not far from $p_{0}$.

For anisotropic average stresses a more general definition of OCR is necessary. For the time being the definition used in the isotropic visco-hypoplastic model of Niemunis [53], which originates from the MCC model, is adopted for the HCA model. The preloading surface is an ellipse described by

$p\left(p-p_{e}^{+}\right)+\left(\frac{q}{M}\right)^{2}=0$

with the critical stress ratio $M$. The equivalent pressure $p_{e}^{+}$ marks the intercept of the preloading surface with the $p$ axis. The overconsolidation ratio is defined as

$\mathrm{OCR}=\frac{p_{e}}{p_{e}^{+}}$

where $p_{e}$ is calculated from the virgin compression line as observed in isotropic compression tests:

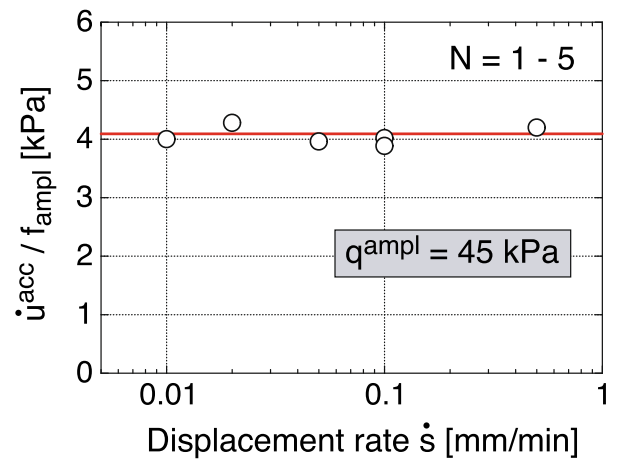

Fig. 6 Inspection of the need of a function $f_{f}$ based on the undrained cyclic tests with different displacement rates $\ln \left(\frac{1+e_{e 0}}{1+e}\right)=\lambda \ln \left(\frac{p_{e}}{p_{e 0}}\right)$

$e_{e 0}$ and $p_{e 0}$ are the referential void ratio and pressure, respectively, and $\lambda$ is the inclination of the compression line in a diagram with log-log scale.

The question, if Eq. (16) should be applied with the actual overconsolidation ratio OCR or its initial value $\mathrm{OCR}_{0}$ is revisited based on element test simulations in Section 4.5.

\subsubsection{Influence of the loading frequency $\left(f_{f}\right)$}

The influence of the loading frequency on the accumulation rate and thus the necessity of a function $f_{f}$ is inspected in Fig. 6. The rates $\dot{u}^{\text {acc }}$ from the initial phase of the tests with different displacement rates performed with $p_{0}=200 \mathrm{kPa}$, $\eta_{0}=0$ and $q^{\text {ampl }}=45 \mathrm{kPa}$ are plotted versus the displacement rates. The $u^{\text {acc }}$ data have been divided by the amplitude function $f_{\text {ampl }}$ (known from Fig. 2) in order to purify them from the influence of different strain amplitudes. No clear dependence of $u^{\text {acc }} / f_{\text {ampl }}$ on the displacement rate and thus on the loading frequency can be detected in Fig. 6. Therefore, the respective function can be set to $f_{f}=1$ for kaolin. For materials with higher plasticity subjected to cyclic loading such as reported in [75], however, a function $f_{f}$ describing an increase in the cumulative rates with decreasing loading frequency will be indispensable.

\subsubsection{Influence of the loading direction with respect to the sedimentation direction}

Figure 7 compares the $u^{\text {acc }}$ data for samples cut out in the horizontal or in the vertical direction, which were tested with $p_{0}=200 \mathrm{kPa}, \eta_{0}=0$ and $q^{\mathrm{ampl}}=45 \mathrm{kPa}$ or $p_{0}=200$

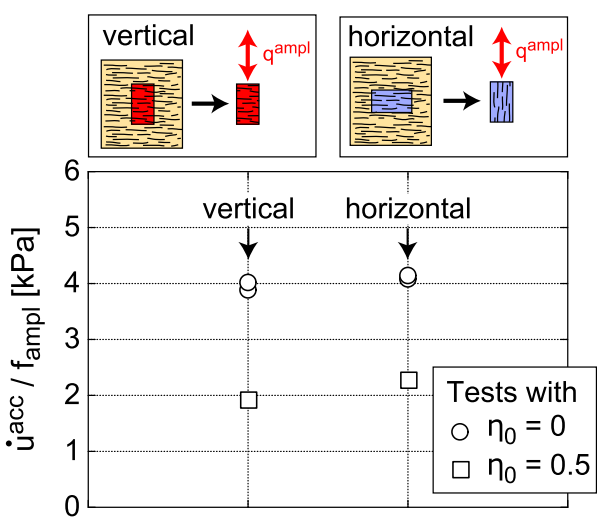

Fig. 7 Comparison of normalized pore pressure accumulation rates $\dot{u}^{\text {acc }} / f_{\text {ampl }}$ from tests on triaxial samples cut out vertically or horizontally from the pre-consolidated kaolin 
Table 1 Functions, material constants and reference quantities for the intensity of accumulation $\dot{\varepsilon}^{\text {acc }}$ according to Eq. (3) (note that the definition of $\dot{f_{N}}$ holds only for $f_{\mathrm{ampl}}=$ const .)

\begin{tabular}{lll}
\hline Function & $\begin{array}{l}\text { Material } \\
\text { constant }\end{array}$ & $\begin{array}{l}\text { Ref. } \\
\text { quant. }\end{array}$ \\
\hline$f_{\mathrm{ampl}}=\left(\varepsilon^{\mathrm{ampl}} / \varepsilon_{\mathrm{ref}}^{\mathrm{ampl}}\right)^{C_{\mathrm{ampl}}}$ & $C_{\mathrm{ampl}}$ & $\varepsilon_{\mathrm{ref}}^{\mathrm{ampl}}$ \\
$\dot{f_{N}}=C_{N 1} C_{N 2} \exp \left[-\frac{g^{A}}{C_{N 1} f_{\mathrm{ampl}}}\right]+C_{N 1} C_{N 3}$ & $\begin{array}{l}C_{N 1}, C_{N 2}, \\
C_{N 3}\end{array}$ \\
$f_{e}=\frac{\left(C_{e}-e\right)^{2}}{1+e} \frac{1+e_{\mathrm{ref}}}{\left(C_{e}-e_{\mathrm{ref}}\right)^{2}}$ & $C_{e}$ & $e_{\mathrm{ref}}$ \\
$f_{\eta}=\exp \left(C_{\eta}\left|\eta^{\mathrm{av}}\right| / M\right)$ & & \\
$f_{O C R}=\exp \left[-C_{\mathrm{OCR}}(\mathrm{OCR}-1)\right]$ & $C_{\eta}$ & $M$ \\
$f_{f}=1$ & $C_{\mathrm{OCR}}$ & \\
\hline
\end{tabular}

$\mathrm{kPa}, \eta_{0}=0.5$ and $q^{\mathrm{ampl}}=30 \mathrm{kPa}$. After the influence of strain amplitude has been eliminated by the division through $f_{\text {ampl }}$, the data show almost no influence of the cutting direction. Therefore, the effect of anisotropy needs not be considered in Eq. (3). It should be captured, however, in the low-cycle model used to evaluate the field of the strain amplitude. In the present case the AVISA model is used for that purpose.

\subsection{Direction of accumulation}

The definition of the direction of accumulation $\mathbf{m}$ of the MCC model is also used for the HCA model for clay:

$\mathbf{m}=\left[\frac{1}{3}\left(p^{\mathrm{av}}-\frac{\left(q^{\mathrm{av}}\right)^{2}}{M^{2} p^{\mathrm{av}}}\right) \mathbf{1}+\frac{3}{M^{2}}\left(\boldsymbol{\sigma}^{\mathrm{av}}\right)^{*}\right]$

where $\sqcup \rightarrow=\sqcup /\|\sqcup\|$ denotes the normalization of a tensorial quantity and $\sqcup^{*}=\sqcup-\operatorname{tr}(\sqcup) / 31$ denotes the deviatoric part of a tensor. $\mathbf{1}$ is the second-order unit tensor. The suitability of this definition for $\mathbf{m}$ is discussed in $[78,80]$. Therein an alternative anisotropic definition of $\mathbf{m}$ is investigated as well. It was concluded that the isotropic definition of $\mathbf{m}$ is sufficient.

\subsection{Incorporation of plastic strain and hypo- elastic stiffness}

Analogous to the HCA model for sand, incorporation of a plastic strain rate is required since the stress can take unphysical values otherwise (e.g. far outside the failure locus). The isotropic yield surface of the MCC model introduced in Eq. (17) is used for this purpose. Using an associative flow rule, the plastic strain can be calculated by

$$
\dot{\boldsymbol{\varepsilon}}^{\mathrm{pl}}=\dot{\phi} \frac{\partial F}{\partial \boldsymbol{\sigma}}
$$

where $\dot{\phi}>0$ is the rate of plastic potential and $F$ is the scalar value of the yield surface in Eq. (17). $\frac{\partial F}{\partial \sigma}$ is equivalent to $\mathbf{m}$ introduced in Eq. (20). The plastic strain is calculated iteratively using Newton's method. The mathematical procedures are described in Appendix A.

A simple isotropic stiffness is used for $E$ in Eq. (1)

$\mathrm{E}=K \mathbf{1} \otimes \mathbf{1}+2 \mu \mathrm{l}$

where the scalar factors are defined by:

$K=\frac{1+e}{\kappa} p$ and $\mu=\frac{3 K(1-2 v)}{2(1+v)}$

In Eq. (23) $\kappa$ is the swelling index and $v$ is the Poisson's ratio. I in Eq. (22) is the symmetric fourth-order unit tensor. As has been outlined in [55], a hyper-elastic definition of stiffness is inessential for the HCA model since such models do not describe the course of stress and strain during individual cycles. Thus, no accumulation of artificial strain during closed stress cycles or vice versa occurs despite the hypo-elastic definition of stiffness.

\subsection{Final set of equations and calibration of the parameters}

The definition of the functions of the factors $f_{\sqcup}$ used for the calculation of the intensity of strain accumulation (Eq. (3)) and their parameters are summarized in Table 1 . The model needs a total of 7 material constants and 2 reference quantities (apart from the parameters for the direction of accumulation and elastic stiffness). For the calibration, undrained cyclic triaxial tests with variations of

Table 2 HCA model parameters for kaolin $\left(\varepsilon_{\mathrm{ref}}^{\mathrm{ampl}}=10^{-3}, e_{\text {ref }}=1.25\right)$, for different OCR approaches used in function $f_{\mathrm{OCR}}$

\begin{tabular}{llllllll}
\hline$f_{\mathrm{OCR}}$ & $C_{\mathrm{ampl}}$ & $C_{N 1}$ & $C_{N 2}$ & $C_{N 3}$ & $C_{e}$ & $C_{\eta}$ & $C_{\mathrm{OCR}}$ \\
\hline $\mathrm{OCR}_{0}$ & 0.6 & 0.00115 & 0.8 & 0.0 & -0.97 & 2.9 & 0.5 \\
$\mathrm{OCR}$ & 0.8 & 0.00125 & 0.5 & 0.0 & -0.97 & 2.9 & 0.5 \\
\hline
\end{tabular}


Simulations
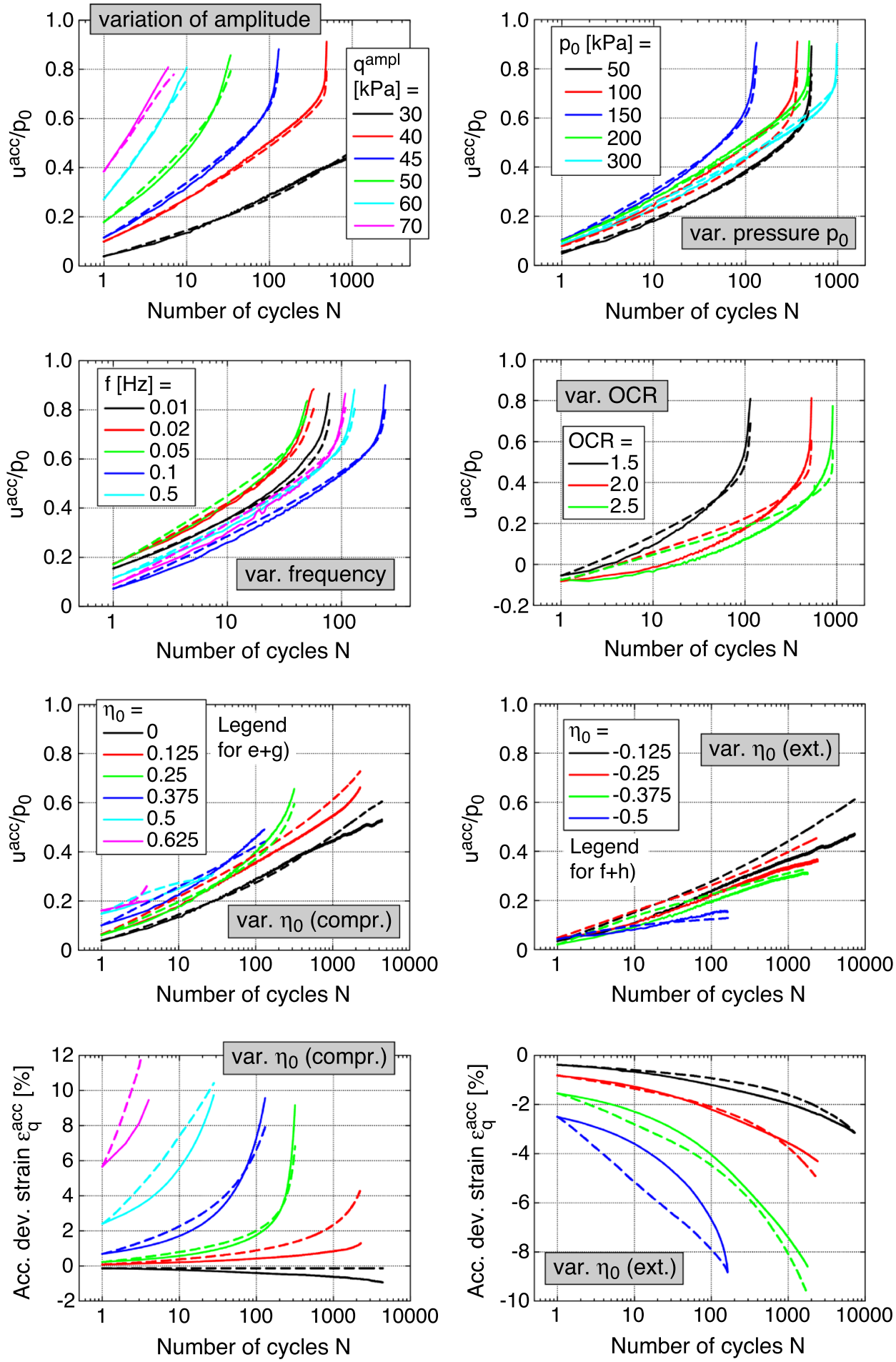

Fig. 8 Comparison of measured and simulated pore pressure accumulation $\left(u^{\text {acc }}(N)\right)$ and deviatoric strain accumulation $\left(\varepsilon_{q}^{\text {acc }}(N)\right)$ curves. The simulations have been performed applying a variable OCR 
- $\quad$ stress amplitude (parameter $C_{\text {ampl }}$ )

- initial void ratio (parameter $C_{e}$ )

- average stress ratio (parameter $C_{\eta}$ )

- initial overconsolidation ratio (parameter $C_{\mathrm{OCR}}$ )

are necessary while keeping the remaining parameters constant within each series. The parameters $C_{N 1}, C_{N 2}$ and $C_{N 3}$ can be finally determined based on the data from all tests.

\subsection{Element test simulations}

All cyclic tests performed on kaolin have been simulated with an element test program. It calculates the curves of accumulated pore water pressure $u^{\text {acc }}(N)$ and accumulated deviatoric strain $\varepsilon_{q}^{\text {acc }}(N)$, using the initial stress $\left(p_{0}, \eta_{0}\right)$, the initial void ratio $e_{0}$, the preloading pressure $p_{\text {preload }}$ and the measured strain amplitudes $\varepsilon^{\text {ampl }}(N)$ (changing from cycle to cycle) as input. The overconsolidation ratio entering $f_{\mathrm{OCR}}$ was either evaluated as $\mathrm{OCR}=p_{e} / p_{e}^{+}$with $p_{e}^{+}$continuously updated for the actual stress state or simply set to the initial value $\mathrm{OCR}_{0}$. In the first case, OCR increases during the undrained cyclic tests also for normally consolidated samples, due to the reduction in $p_{e}^{+}$. The latter approach leads to $\mathrm{OCR}=1$ for normally consolidated samples throughout the test.

Starting from the values estimated from Figs. 2, 3, 4 and 5 , the parameters of the intensity functions in Table 1 have been iteratively optimized in the element test simulations. The optimized parameters are summarized in Table 2 .

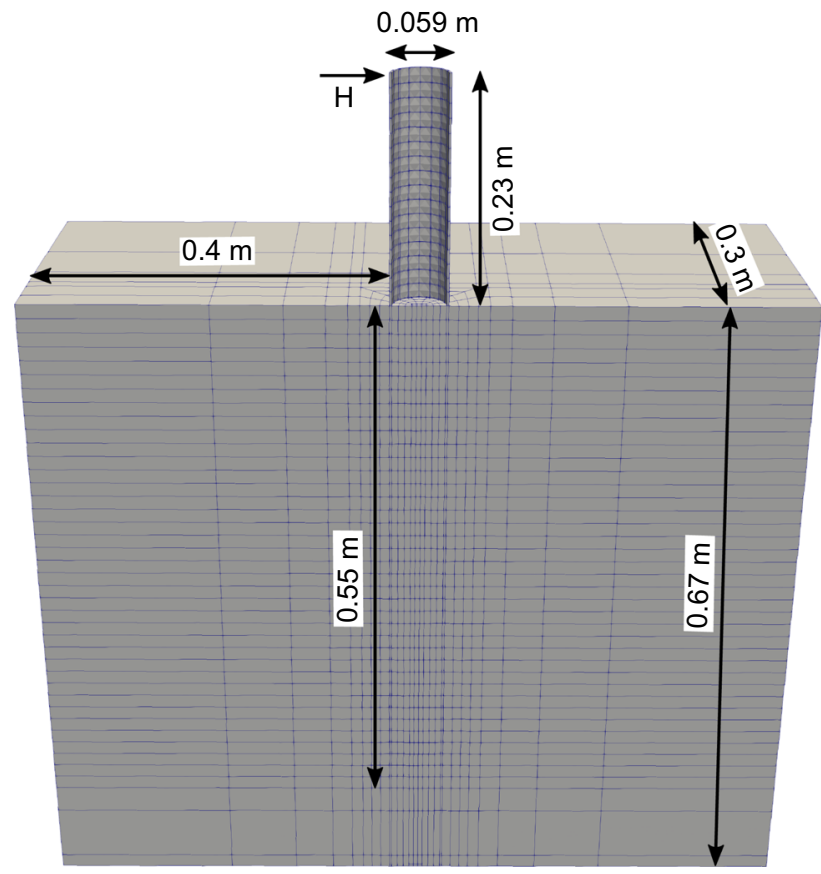

Fig. 9 Finite element model of the centrifuge test. Note that only exterior nodes are displayed.
Despite Fig. 4 , the same value $C_{\eta}$ has been applied for the triaxial compression and extension tests. The curves $u^{\mathrm{acc}}(N)$ and $\varepsilon_{q}^{\mathrm{acc}}(N)$ obtained from the simulations with the variable OCR are compared to the experimental data in Fig. 8. With the exception of some discrepancies for single tests, a satisfying reproduction of the data from the cyclic laboratory tests can be concluded. The results look similar for the approach assuming a constant OCR (OCR = $\mathrm{OCR}_{0}$ ). However, different material constants are needed for the different approaches. If the actual OCR is applied in $f_{\mathrm{OCR}}$, the increasing OCR values lead to a decrease in $f_{\mathrm{OCR}}$ with $N$ and thus the predicted cumulative rates $u^{\text {acc }}$ and $\dot{\varepsilon}_{q}^{\text {acc }}$ get lower. This can be compensated by larger parameters $C_{\text {ampl }}$ and $C_{N 1}$, while $C_{N 2}$ has to be reduced (Table 2).

\section{Back-analysis of centrifuge tests on monopiles in soft clay}

In order to show the feasibility of the proposed constitutive model for the study of OWT foundations subjected to lateral cyclic loading, a back-analysis of centrifuge tests performed by Yang et al. [85] on soft kaolin clay is presented in the following. Note that a back-analysis of these tests has been presented in [20] as well, using an elastoplastic bounding surface model and assuming locally undrained conditions.

\subsection{Centrifuge tests by Yang et al. (2019)}

The considered centrifuge tests have been performed at the Zhejiang University in 2019. All quantities are given in model scale in the following. A strongbox with internal dimensions of $1.2 \mathrm{~m} \times 0.9 \mathrm{~m} \times 1.0 \mathrm{~m}$ and Malaysia kaolin clay (plastic limit $w_{P}=35 \%$ and liquid limit $w_{L}=80 \%$ ), which is a standard material of the National University of Singapore, have been used.

The soil sample was prepared by mixing dry kaolin powder with water using a soil-mixing machine and subjecting the slurry to 8 hours of consolidation in the centrifuge at $100 \mathrm{~g}$. For these tests, water and not a fluid with a higher viscosity has been used as pore fluid. Following the consolidation of the clay, the centrifuge was stopped and the pile was pushed into the soil. The aluminium pile had an outer diameter of $0.059 \mathrm{~m}$, a wall thickness of $0.0022 \mathrm{~m}$ and an embedded length of $0.55 \mathrm{~m}$. Once the pile had been installed, the soil including the pile was subjected to $100 \mathrm{~g}$ for one hour in order to allow for any settlement around the pile.

Following the resting period, the pile was laterally loaded at $0.23 \mathrm{~m}$ above the ground surface. Three cyclic load packages with varying amplitude and average loading with 
Table 3 Parameters of the AVISA model for Malaysian kaolin clay used in the FE simulations of the centrifuge tests

\begin{tabular}{|c|c|c|c|c|c|c|c|c|c|c|c|c|c|c|}
\hline $\begin{array}{l}\lambda \\
{[-]}\end{array}$ & $\begin{array}{l}\kappa \\
{[-]}\end{array}$ & $\begin{array}{l}v_{h} \\
{[-]}\end{array}$ & $\begin{array}{l}\alpha \\
{[-]}\end{array}$ & $\begin{array}{l}M_{c} \\
{[-]}\end{array}$ & $\begin{array}{l}e_{i 0} \\
{[-]}\end{array}$ & $\begin{array}{c}f_{b 0} \\
{[-]}\end{array}$ & $\begin{array}{l}I_{v} \\
{[-]}\end{array}$ & $\begin{array}{l}R \\
{[-]}\end{array}$ & $\begin{array}{l}m_{R} \\
{[-]}\end{array}$ & $\begin{array}{l}d \\
{[-]}\end{array}$ & $\begin{array}{l}\beta_{0} \\
{[-]}\end{array}$ & $\begin{array}{l}\chi_{0} \\
{[-]}\end{array}$ & $\begin{array}{l}\chi_{\max } \\
{[-]}\end{array}$ & $\begin{array}{c}C_{a} \\
{[-]}\end{array}$ \\
\hline 0.244 & 0.053 & 0.25 & 1.2 & 0.9 & 2.34 & 2 & 0.015 & $10^{-4}$ & 5 & 3 & 0.1 & 5 & 30 & 0.005 \\
\hline
\end{tabular}

100 cycles each and a frequency of $0.2 \mathrm{~Hz}$ have then been applied. $30 \mathrm{~s}$ of consolidation between the packages has been considered. Only the first two cyclic loading packages are considered for the back-analysis since the third package with the largest amplitudes led to large pile head displacements (approximately $1 \mathrm{~m}$ in prototype scale) for which a large-deformation numerical method should be applied.

\subsection{Numerical model of the centrifuge tests}

The finite-element analysis was performed using the program numgeo written by the first two authors (Machaček $\&$ Staubach, see $[43,45,63,69]$ and numgeo.de). numgeo is a finite-element code specialized on geotechnical boundary value problems. numgeo has been applied for the simulation of vibratory pile driving in saturated sand reported in $[45,65]$ and for the investigation of the influence of the installation on the behaviour of piles subjected to lateral cyclic loading using the HCA model for sand in [63].

The finite element model of the centrifuge test is displayed in Fig. 9. The simulations were performed in model scale. In order to incorporate the change in pore water pressure as well as consolidation effects, u-p elements were used. $u-p$ elements discretize the displacement of the solid phase $\boldsymbol{u}$ and the pore water pressure $p^{w}$. As has been shown in [62], u-U elements (discretizing the displacement of solid phase $\boldsymbol{u}$ and the displacement of water $\boldsymbol{u}^{w}$ ) could be advantageous in case of low hydraulic conductivities and small time increments since the matrix conditioning of the resulting left-hand side is superior to the one of u-p elements. The well-known oscillations in pore water pressure occurring when using u-p elements are absent using u-U elements. However, since the investigation of the constitutive model is the main target in the current study, the 'conventional' u-p elements are judged to be sufficient for the present purpose. $u 27 p 8$ brick elements, with 27 nodes discretizing the displacement using bi-quadratic Lagrangian interpolation functions and 8 nodes discretizing the pore water pressure using linear interpolation functions, were utilized.

A surface-to-surface (STS) method was used for the contact discretization. Using the STS technique, the contact stress is determined in each contact surface integration point by calculating the shortest distance to the paired surface by evaluation of the convective coordinate. Due to the bi-quadratic shape functions of the $u 27 p 8$ element the derivatives are continuous and the normal vector is definable at every point of the surface. Details on the employed STS technique can be found in [67]. A stiffness-dependent penalty factor (30 times the trace of the stiffness tensor of the adjacent continuum) was used to enforce the normal contact constraints. A Coulomb friction model with a friction coefficient of 0.2 was applied.

A bulk modulus of the pore water of $2.2 \mathrm{GPa}$ was assumed. The permeability of the Malaysian kaolin clay is $K^{\text {Perm }}=\eta^{w} k^{w} / \gamma^{w}=2 \cdot 10^{-15} \mathrm{~m}^{2}$ (assuming the dynamic viscosity of water to be $\left.\eta^{w}=1 \cdot 10^{-6} \mathrm{kPas}\right)$. With the increased gravity in the centrifuge test, this resulted in a hydraulic conductivity of $k^{w} \approx 2 \cdot 10^{-6} \mathrm{~m} / \mathrm{s}$ during the testing.

Installation effects were not taken into account in the simulations since in the experiment the pile has been jacked into the soil at $1 \mathrm{~g}$. Due to the increase in gravity to $100 \mathrm{~g}$, the influence of the installation on the initial soil state is assumed to decrease significantly. The initial overconsolidation ratio prior to the spin-up of the centrifuge was assumed to be one. The lateral stress coefficient was set to 0.5 .

For the low-cycle part of the simulations, the anisotropic visco-ISA (AVISA) model [73] was used. This model is a rate-dependent model for fine-grained soils able to reproduce small-strain effects, inherent anisotropy as well as the influence of the overconsolidation ratio. The strain rate dependency is reproduced by incorporating a third strain rate mechanism (in addition to the elastic and hypoplastic strain rate), which can be switched off in case of lowplasticity fine-grained soils. To cover a wide range of strain/stress amplitudes as well as to account for the specific behaviour of clays the ISA plasticity approach of [24] has been revised and slightly reformulated in [73]. Furthermore, to account for initially anisotropically consolidated states (induced anisotropy) the kinematic hardening mechanism of [24] has been modified according to the experimental findings gained in [78]. In addition, a loading surface has been incorporated to define a threedimensional overconsolidation ratio and to account for its effects on the accumulation behaviour. To capture the inherent anisotropy exhibited by some clays, a transversal 
hypoelastic stiffness has been further introduced into the mathematical formulation of AVISA. The model is also able to reproduce the cyclic tests presented in Figure 8 well, as shown in [73], and is thus technically applicable for the complete simulation of the centrifuge tests (without the HCA model). However, the computational effort necessary to simulate all cycles conventionally exceeded the available computational resources. Therefore, the application of the HCA model seems compulsory for the present simulations.

The parameters of the AVISA model have been partially determined for the Malaysian kaolin clay $\left(w_{P}=\right.$ $35 \%, w_{L}=80 \%$ ) used in the centrifuge tests (parameters of the MCC model: $\lambda, \kappa, v_{h}$ and $M_{c}$ obtained from [42]) or have been assumed to lie between the parameters calibrated for the kaolin clay considered in the previous sections $\left(w_{P}=12.2 \%, w_{L}=47.2 \%\right)$ and those for the more plastic Lower Rhine Clay $\left(w_{P}=34 \%, w_{L}=56.1 \%\right)$ reported in $[71,73]$. The utilized set of parameters is given in Table 3 , whereby further details about the correlations used for the calibration as well as the numerical calculation of an oedometric compression test are provided in Appendix B. It is worth mentioning that using the HCA model for clay, the influence of the conventional model is much lower than in case of the HCA model for sand since the influence of the strain amplitude, calculated by the intrinsic model, is much lower (see lower $C_{\text {ampl }}$ values discussed in Section 4.1.1).

The soil inside the pile has been modelled elastically since it is only involved in a rigid deformation when the pile rotates and does not influence the pile response. Preliminary simulations showed that an elastic stiffness of $10,000 \mathrm{kPa}$ and a Poisson's ratio of 0.3 are appropriate values.

The parameters of the HCA model for clay determined in the previous Section given in Table 2 (MCC flow rule with $\mathrm{OCR}_{0}$ ) were applied for the simulations. The same reference values were used as well. For the calculation of $f_{\eta}$ and the direction of accumulation the critical friction angle used in Sect. 4.1.4 was employed.

During the high-cycle phase of the HCA model, instead of introducing update cycles as shown in Figure 1, the change of the strain amplitude $\varepsilon^{\text {ampl }}$ due to the change in the soil stiffness caused by the cyclic loading has been taken into account using a so-called adaptive strain amplitude definition, which has been recently proposed in [66]. The strain amplitude is periodically updated by linearly scaling it with respect to the change in soil stiffness compared to the stiffness at the end of the second load cycle. A non-local smoothing algorithm is used to avoid localization of large values of strain amplitude in some integration points due to self-reinforcement effects similar to the approaches used in non-local plasticity. For a detailed description of this procedure, the interested reader is referred to [66].

The simulations were performed in the following steps:

1. Application of the self-weight of the soil and pile.

2. Application of the average value of the lateral force $H^{\text {av }}=62.5 \mathrm{~N}$ linearly increasing over a time span of $3 \mathrm{~s}$.

3. Calculation of the first cycle, using the AVISA model. The average load $H^{\text {av }}$ was superposed by a sinusoidal cyclic load with the amplitude $H^{\text {ampl }}=37.5 \mathrm{~N}$.

4. Calculation of the second cycle, using the AVISA model. During this second cycle, the strain path was recorded in each integration point. The spatial field of the strain amplitude $\varepsilon^{\text {ampl }}$ was determined from that strain path. It was used as input for the function $f_{\text {ampl }}$ in the following calculation with the HCA model. During the high-cyclic loading, the strain amplitude was updated every 10th calculation increment (approximately at $N=3,8,15,40,90)$.

5. Calculation of permanent deformations due to $N=100$ further cycles using the HCA model. The load was kept constant at its average value $H^{\text {av }}$ while the permanent
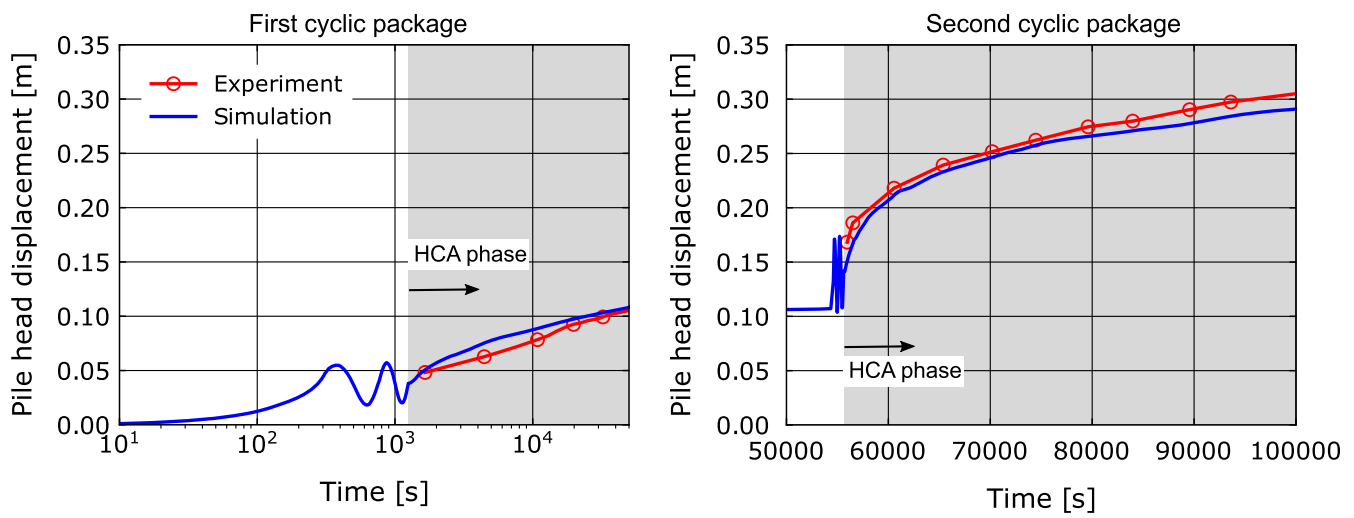

Fig. 10 Displacement of the pile head versus time for the measurements and the results of the simulation, respectively 


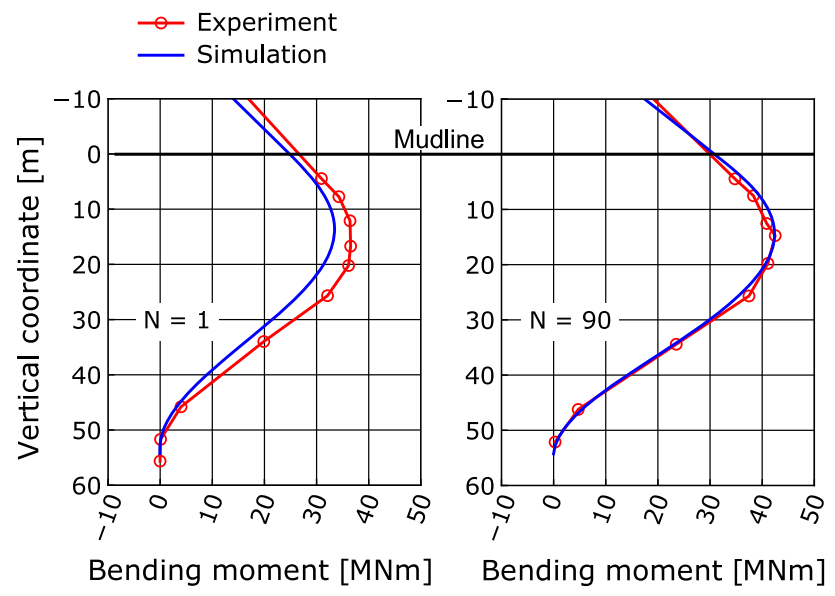

Fig. 11 Comparison of the bending moment along the pile between the measurements made in the experiment and the results of the simulations after $N=1$ and $N=90$ cycles of the first load package

deformations due to the cyclic loading were predicted by the HCA model. Taking into account the frequency of the cyclic loading of $0.2 \mathrm{~Hz}, 500 \mathrm{~s}$ were simulated in the high-cycle phase.

6. $30 \mathrm{~s}$ of consolidation were allowed before application of the next cyclic package. Only $H^{\text {av }}$ has been applied in this phase.

7. Repetition of steps 3-6 using the load magnitudes of the second package of cyclic loads $\left(H^{\mathrm{av}}=110 \mathrm{~N}\right.$ and $H^{\mathrm{ampl}}=65 \mathrm{~N}$ ).

\subsection{Results of the back-analysis}

As has been done in the study by Yang et al. [85], the results are evaluated in prototype scale. The pile head displacement measured at the point of load application is given in Figure 10 for the measurements as well as for the results of the simulation. The results of the experiment are displayed as mean pile head displacement beginning after the end of the second load cycle. The measured displacement after the first two cycles is slightly underestimated by
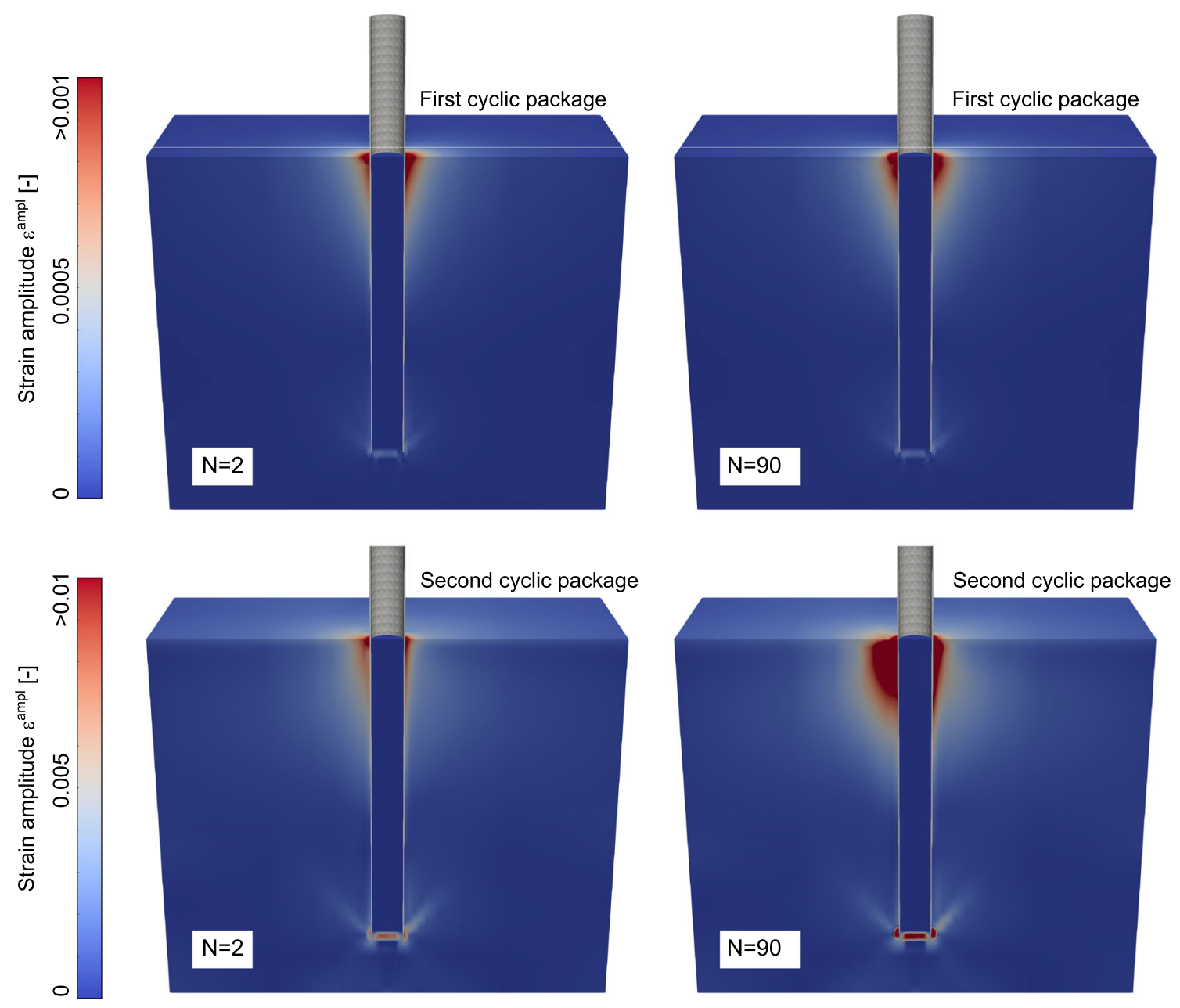

Fig. 12 Spatial distribution of the strain amplitude at $N=2$ and $N=90$ for the first cyclic load package (top part of figure) and the second package (lower part of figure). Note the different scale of the legends 


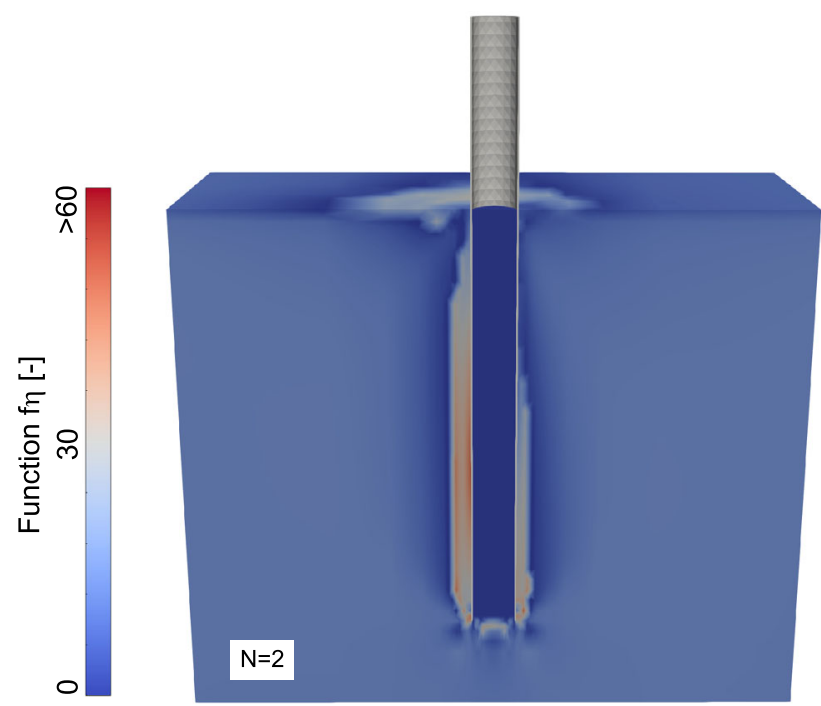

Fig. 13 Spatial distribution of the function $f_{\eta}$ directly at the start of the HCA phase $(N=2)$ of the first cyclic load package

the simulation using the AVISA model, which yields approximately $0.04 \mathrm{~m}$. The subsequent HCA phase starts at $t=1,300 \mathrm{~s}(N=2)$ and first predicts a very fast accumulation which slows down after approximately $\approx 1,500$ s. Compared to the experiment the accumulation rate of permanent pile head displacement is slightly too small in this latter phase. The pile head displacement predicted at the end of the first cyclic load package fits well to the measurements, however.

For the second load package two individual cycles are simulated again using AVISA. Due to the larger loading magnitudes, larger displacement amplitudes are observed compared to the cyclic loading of the first package (see plot on right-hand side of Fig. 10). Consequently, the strain amplitude is larger leading to a higher accumulation rate of the HCA model in the subsequent high-cycle phase. The accumulation of pile head displacement predicted by the HCA model during this phase fits well to the values recorded in the experiment.

The curves of bending moment versus the height of the pile after $N=1$ and $N=90$ cycles are given in Figure 11 . The mudline is located at a vertical coordinate of $0 \mathrm{~m}$. The distribution after one cycle shows that the simulation underestimates the magnitude of the bending moment slightly but captures the general shape of the measured curve very well. After the application of 90 cycles both the bending moment measured in the experiment and the one obtained from the simulation have increased in magnitude. This increase is more pronounced in case of the simulation. The accordance at $N=90$ between the measurements and the results of the simulation is almost perfect.

Figure 12 displays the spatial distribution of the strain amplitude $\varepsilon^{\text {ampl }}$ at $N=2$ and $N=90$ for the first cyclic

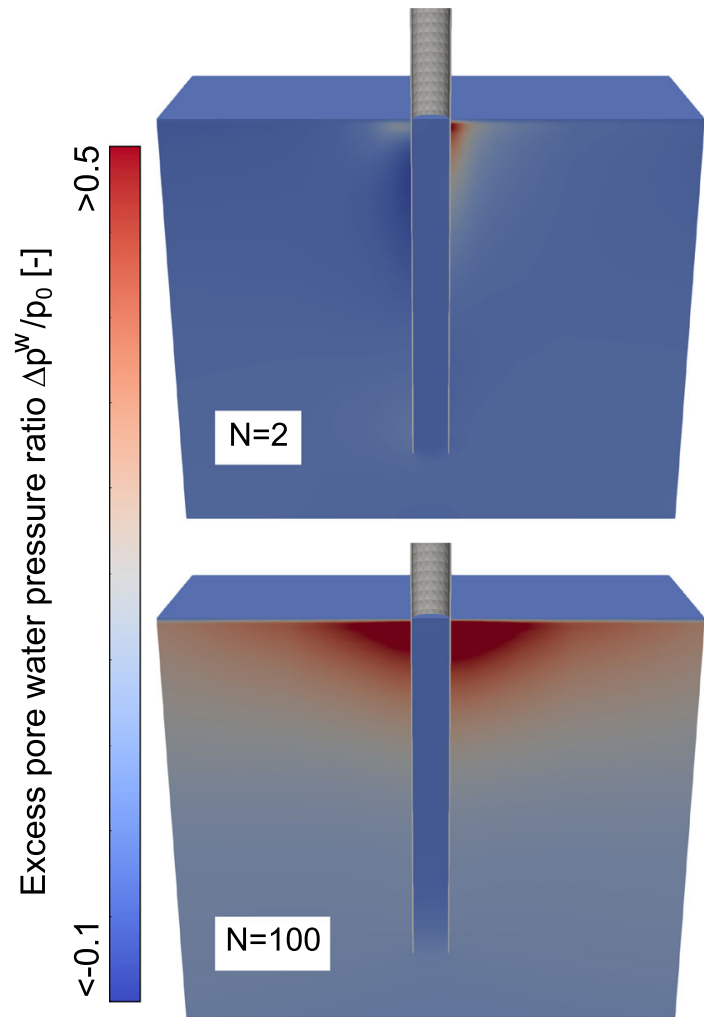

Fig. 14 Spatial distribution of the excess pore water pressure ratio at $N=2$ (top part of figure) and at the end of the HCA phase at $N=100$ cycles (lower part of figure) for the first cyclic load package

load package (top part of figure). The largest strain amplitudes with values above $0.1 \%$ occur at the soil surface in the vicinity of the pile. Thus, the function $f_{\text {ampl }}$ is largest at this location. As has been explained earlier, the change in the strain amplitude is taken into account using an adaptive strain amplitude definition. The spatial distribution of the strain amplitude following the last update performed during the first cyclic load package (at $N=90$ ) shows that the strain amplitude has changed only slightly due to the application of 90 cycles. Moderately larger values are obtained at the left-hand side of the pile (the average loading of the pile is applied to the right) compared to the distribution at $N=2$. The strain amplitude is much larger for the second cyclic load package as is visible from the lower part of Fig. 12. During the second cyclic load package, the change of the strain amplitude is larger compared to the first cyclic load package as is visible from the distribution at $N=90$. Therefore, the consideration of the change in strain amplitude in the simulation with the HCA model is judged mandatory for the cyclic loading with larger amplitudes but is of less importance for the cyclic load package with lower load magnitude. This is reasonable since the cyclic loading with higher magnitude causes larger increase in excess pore water pressure and 


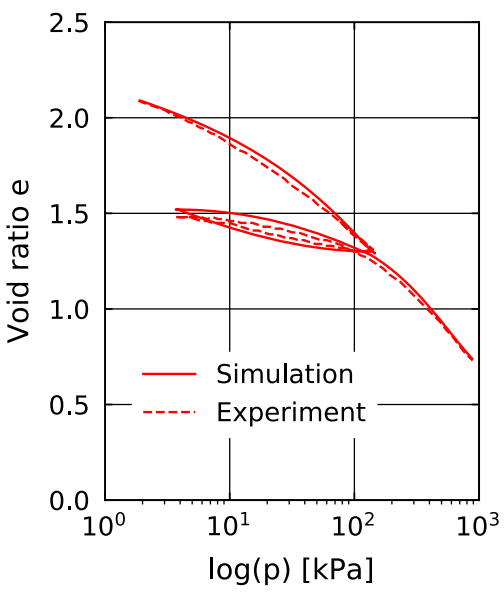

Fig. $15 e-\log (p)$ results of an oedometric compression test on Malaysia kaolin clay from [42] and simulation with the AVISA model

consequently a more pronounced reduction in soil stiffness leading to larger strain amplitudes.

The function $f_{\eta}$ shows large values alongside the lefthand side of the pile at the start of the HCA phase as is visible from Fig. 13. Since the pile is loaded to the right, the horizontal effective stress is reduced on its left, which leads to large average stress ratios $\eta^{\text {av }}$. From the factors influencing the rate of strain accumulation, $f_{\eta}$ varies the most in magnitude over the calculated domain. While the factor $f_{e}$ takes values in the range of 0.8 to $1.3, f_{\eta}$ varies from 0 to values above 60. $f_{\text {ampl }}$ has a comparably modest influence with values in the range of 0 to 2 (for the first cyclic load package). $f_{\mathrm{OCR}}$ takes values reaching from 1 to 2 due to the change in the overconsolidation ratio prior to the HCA phase caused by the first two cycles of loading.

The fields of excess pore water pressure ratio $\left(\Delta p^{w} / p_{0}\right)$ at the beginning of the HCA phase (at $N=2$ ) and at the end of the simulation (at $N=100$ ) are given in Figure 14 for the first cyclic load package. At $N=2$ an increased pore water pressure in the upper half of the soil on the right-hand side of the pile (where the soil is pushed by the pile) is evident, while a decrease is observed in the soil on the left-hand side (where the soil is unloaded). After the application of 100 cycles, a significant increase in the soil area at the upper part of the pile affected by large excess pore water pressures is observed. The increase in excess pore water pressure is due to the tendency of the soil to compact around the pile when subjected to cyclic loading. The consolidation process taking place simultaneously is, however, not fast enough to allow free drainage of the pore water.

\section{Summary, conclusions and outlook}

A HCA model for clay which predicts the accumulation of permanent strain under cyclic loading with a large number of cycles has been presented. The data of an extensive laboratory testing program on kaolin clay under undrained cyclic loading has been analysed for this purpose. Compared to sand under cyclic loading, a lower influence of the strain amplitude on the strain accumulation was found. The rates of strain accumulation were found to increase with increasing void ratio and increasing stress ratio. As expected, samples with larger initial overconsolidation ratios showed less accumulation. No significant influence of the frequency of loading on the rate of accumulation was observed for the studied kaolin, due to its rather low plasticity.

Building upon these results, constitutive equations of the HCA model for clay have been proposed. The concept of the constitutive model is based on the HCA model for sand by Niemunis et al. [55]. The model has then been validated by the simulation of element tests and the back-analysis of a centrifuge test on a monopile in soft clay under cyclic lateral loading. The results of the simulation of the centrifuge test are in good accordance with the measured data regarding the development of pile displacement with the number of applied cycles. The measured bending moment of the pile after $N=1$ and $N=90$ cycles could also be captured accurately by the simulation.

Future work will focus on the further development and validation of the constitutive equations for clays with higher plasticity. An influence of the loading frequency on the accumulation rate is expected in this case. In addition, other relations for the overconsolidation ratio as for example the equivalent time line model proposed by [38] could be adopted. In future work it will be investigated whether the incorporation of this concept can cover both the overconsolidation effects as well as the frequency dependence of plastic fine-grained soils. The concept of [38] can even be generalized for three-dimensional stress states considering the formulation developed in e.g. [73] and cover the influence of the stress ratio as well. Thus, the factor $f_{\eta}$ could maybe be omitted. Since the proposed model was developed on the basis of tests on reconstituted samples only, it is intended to study the accumulation behaviour of undisturbed natural samples in future work, where effects of destructuring have to be additionally considered.

The proposed model can also be used for a parametric study on monopile foundations in clay similar to the study considering sandy soil [68] and up to 1 million load cycles. In addition, the influence of the installation of the pile prior to the cyclic lateral loading should be studied. 
Investigations considering sand showed that the installation induced changes in the soil state influence the pile response to cyclic lateral loading considerably [64]. So far, it is not clear how large the installation (method) influences the pile response to subsequent loading in cohesive soils.

\section{Calculation of plastic strain}

The increment in plastic strain is calculated by

$\Delta \varepsilon^{\mathrm{pl}}=\Delta \phi \frac{\partial F}{\partial \boldsymbol{\sigma}}$

using the definition of the yield criterion $F$ given in Eq. (17).

$\Delta \phi$ is determined by computing the zero of Eq. (17) using Newton's method:

$$
\Delta \phi^{i+1}=\Delta \phi^{i}-\frac{F^{i}}{\frac{d F^{i}\left(\Delta \phi^{i}\right)}{d \Delta \phi}}
$$

Equation (25) is solved in every iteration $i$ until $F$ is sufficiently close to zero. Using the chain rule, the derivative of $F$ with respect to $\Delta \phi$ is obtained:

$$
\frac{d F(\Delta \phi)}{d \Delta \phi}=\frac{\partial F}{\partial p} \frac{\partial p}{\partial \Delta \phi}+\frac{\partial F}{\partial q} \frac{\partial q}{\partial \Delta \phi}+\frac{\partial F}{\partial p_{e}^{+}} \frac{\partial p_{e}^{+}}{\partial \Delta \phi}
$$

The partial derivatives of $p, q$ and $p_{e}^{+}$are (see [11]):

$$
\begin{aligned}
\frac{\partial p}{\partial \Delta \phi} & =-K \frac{\left(2 p-p_{e}^{+}\right)}{1+\left(2 K+\vartheta p_{e}^{+}\right) \Delta \phi} \\
\frac{\partial q}{\partial \Delta \phi} & =-\frac{q}{\Delta \phi+M^{2} / 6 \mu} \\
\frac{\partial p_{e}^{+}}{\partial \Delta \phi} & =\vartheta p_{e}^{+} \frac{2 p-p_{e}^{+}}{1+\left(2 K+\vartheta p_{e}^{+}\right) \Delta \phi}
\end{aligned}
$$

In Eq. (29) $\vartheta$ is defined by:

$$
\vartheta=\frac{1+e}{\lambda-\kappa}
$$

\section{Calibration of AVISA parameters}

The AVISA model requires the calibration of 15 parameters:

- Four parameters are needed for the description of the transversal (hypo)elastic stiffness: the compression index $\lambda$, the swelling index $\kappa$, the Poisson's ratio $v_{h}$ and the anisotropic coefficient $\alpha . \lambda, \kappa$ and $v_{h}$ have been adopted from the MCC model parameters reported for Malaysia kaolin clay in [42]. The accuracy of these values for the AVISA model has been proven by simulating the oedometric compression test from [42] with one unloading-reloading cycle, as shown in Fig. 15. $\alpha$ has been chosen to lie between the values reported for the kaolin considered in the present study and the highly plastic Lower Rhine Clay reported in [71].

- For the determination of the critical as well as the loading surface, three parameters are required: the triaxial compression critical state slope $M_{c}=$ $6 \sin \left(\varphi_{c}\right) /\left[3-\sin \left(\varphi_{c}\right)\right]$ adopted from the MCC value reported in [42]; the loading surface factor $f_{b 0}$ determined in the same manner as $\alpha$ and the maximum void ratio $e_{i 0}$ at $p_{\text {ref }}=1 \mathrm{kPa}$ calibrated by means of the empirically derived correlation function $e_{i 0}=0.9 \rho^{s} / \rho^{w}$ [71] with $\rho^{s}=2.6 \mathrm{~g} / \mathrm{cm}^{3}$ from [85].

- One parameter is responsible for the rate dependency of the model, the so-called viscosity index $I_{v}$ defining explicitly the creep intensity. It has been assumed identical to the value calibrated for kaolin clay. However, a more accurate calibration of this parameter could improve significantly the predictive capabilities of the model.

- The seven parameters which define the small-strain stiffness have been assumed in the same manner as $\alpha$. Since only two cycles are simulated with AVISA, these parameters are of secondary importance and could even be switched off due to the hierarchical composition of the model.

Acknowledgements Parts of this research were funded by German Research Council (DFG) in the framework of the project "Behaviour of cohesive soils under high-cyclic loading: Experimental studies and constitutive description" (WI 3180/2-1) and in the framework of the project "Holistic approach for the design of single piles and pile groups under cyclic loading" (WI 1024/37-1). The financial support by DFG is gratefully acknowledged herewith. The tests analysed in this paper have been performed by the technicians $\mathrm{H}$. Borowski and $\mathrm{F}$. Schwab in the laboratory of the Institute of Soil Mechanics and Rock Mechanics at KIT, Karlsruhe.

Funding Open Access funding enabled and organized by Projekt DEAL.

Open Access This article is licensed under a Creative Commons Attribution 4.0 International License, which permits use, sharing, adaptation, distribution and reproduction in any medium or format, as long as you give appropriate credit to the original author(s) and the source, provide a link to the Creative Commons licence, and indicate if changes were made. The images or other third party material in this article are included in the article's Creative Commons licence, unless indicated otherwise in a credit line to the material. If material is not included in the article's Creative Commons licence and your intended use is not permitted by statutory regulation or exceeds the permitted use, you will need to obtain permission directly from the copyright holder. To view a copy of this licence, visit http://creativecommons. org/licenses/by/4.0/. 


\section{References}

1. Andersen KH (2009) Bearing capacity under cyclic loading offshore, along the coast, and on land. The 21st Bjerrum Lecture presented in Oslo, 23 November 2007. Can Geotech J 46(5):513-535

2. Andersen KH (2015) Cyclic soil parameters for offshore foundation design. In: 3rd International Symposium on Frontiers in Offshore Geotechnics (ISFOG), Oslo. Ed. by Meyer, pp 582

3. Andersen KH, Kleven A, Heien D (1988) Cyclic Soil Data for design of gravity structures. J Geotech Eng ASCE 114(5):517-539

4. Andersen KH, Pool JH, Brown SF, Rosenbrand WF (1980) Cyclic and static laboratory tests on Drammen clay. J Geotech Eng Division ASCE 106(GT5):499-513

5. Ansal AM, Erken A (1989) Undrained behavior of clay under cyclic shear stresses. J Geotech Eng ASCE 115(7):968-983

6. API (2007) Recommended Practice for Planning, Designing and Constructing Fixed Offshore Plat-forms-Working Stress Design. 2A-WSD RP2AWSD, Errata and Supplement 3, Washington DC, USA

7. Azzouz AS, Malek AM, Mohsen MB (1989) Cyclic behavior of clays in undrained simple shear. J Geotech Eng ASCE 115(5):637-657

8. Bayat M, Andersen LV, Ibsen LB (2016) P-Y-Y curves for dynamic analysis of offshore wind turbine monopile foundations. Soil Dyn Earthq Eng 90:38-51

9. Bode M, Fellin W, Masin D, Medicus G, Ostermann A (2020) An intergranular strain concept for non-hypoplastic models. Int $\mathbf{J}$ Numer Anal Methods Geomech. 44:1003-1018

10. Borja RI, Kavazanjian E (1985) A constitutive model for the stress-strain-time behaviour of wet clays. Geotechnique 35(3):283-298. https://doi.org/10.1680/geot.1985.35.3.283

11. Borja RI, Lee SR (1990) Cam-Clay plasticity, Part 1: implicit integration of elasto-plastic constitutive relations. Comput Methods Appl Mech Eng 78(1):49-72

12. Boulanger RW, Idriss IM (2007) Evaluation of cyclic softening in silts and clays. Soils Found 133(6):641-652

13. Boulanger RW, Idriss IM (2006) Liquefaction susceptibility criteria for silts and clays. J Geotech Geoenviron Eng ASCE 132(11): 14131428

14. Boulanger RW, Meyers MW, Mejia LH, Idriss IM (1998) Behavior of a fine-grained soil during the Loma Prieta earthquake. Can Geotech J 35(1):146-158

15. Burland JB, Simpson B, St. John HD (1979) Movements around excavations in London clay. In: Design parameters in geotechnical engineering. Proc. 7th European conference on soil mechanics and foundation engineering, Brighton, 1979. Vol. 1, (British Geotechnical Society; distributed by T. Telford, London. Vol. 1. London, UK, pp. 13-29

16. Byrne BW, McAdam RA, Burd HJ, Beuckelaers WJ, Gavin KG, Houlsby GT, Igoe DJ, Jardine RJ, Martin CM, Muirwood A, Potts DM, Gretlund JS, Taborda DM, Zdravkovic L (2020) Monotonic laterally loaded pile testing in a stiff glacial clay till at Cowden. Geotechnique 70(11):970-985

17. Cai Y, Gu C, Wang J, Juang CH, Xu C, Xu H (2013) One-way cyclic triaxial behavior of saturated clay: comparison between constant and variable confining pressure. J Geotech Geoenviron Eng ASCE 139(5):797-809

18. Cai Y, Wu T, Guo L, Wang J (2018) Stiffness degradation and plastic strain accumulation of clay under cyclic load with principal stress rotation and deviatoric stress variation. J Geotech Geoenviron Eng 144(5):04018021

19. Chen C, Xu G, Zhou Z, Kong L, Zhang X, Yin S (2020) Undrained dynamic behaviour of peaty organic soil under long- term cyclic loading, Part II: Constitutive model and simulation. Soil Dyn Earthq Eng, 129

20. Cheng X, Wang T, Zhang J, Liu Z, Cheng W (2021) Finite element analysis of cyclic lateral responses for large diameter monopiles in clays under different loading patterns. Comput Geotech 134:104104

21. Chu DB, Stewart JP, Boulanger RW, Lin PS (2008) Cyclic softening of low-plasticity clay and its effect on seismic foundation performance. J Geotech Geoenviron Eng 134(11):1595-1608

22. Dunnavant TW, O'neill MW (1989) Experimental P-Y Model For Submerged, Stiff Clay. J Geotech Eng, 115(1): 95-114

23. Duque J, Ochmanski M, Masin D, Hong Y, Wang L (2021) On the behavior of monopiles subjected to multiple episodes of cyclic loading and reconsolidation in cohesive soils. Comput Geotech 134:104049

24. Fuentes W, Triantafyllidis T (2015) ISA model: A constitutive model for soils with yield surface in the intergranular strain space. Int J Numer Anal Meth Geomech 39(11):1235-1254. https://doi.org/10.1002/nag.2370

25. Fuentes W, Tafili M, Triantafyllidis T (2018) An ISA-plasticitybased model for viscous and non-viscous clays. Acta Geotechnica 13(2):367-386

26. Fuentes W, Masin D, Duque J (2021) Constitutive model for monotonic and cyclic loading on anisotropic clays. Geotechnique 71(8):657-673

27. Gratchev IB, Sassa K, Fukuoka H (2006) How Reliable is the plasticity index for estimating the liquefaction potential of clayey sands? J Geotech Geoenviron Eng ASCE 132(1):124-127

28. Gu C, Wang J, Cai Y, Yang Z, Gao Y (2012) Undrained cyclic triaxial behavior of saturated clays under variable confining pressure. Soil Dyn Earthq Eng 40:118-128

29. Guo L, Wang J, Cai Y, Liu H, Gao Y, Sun H (2013) Undrained deformation behavior of saturated soft clay under long-term cyclic loading. Soil Dyn Earthq Eng 50:28-37

30. Hanna AM, Javed K (2014) Experimental investigation of foundations on sensitive clay subjected to cyclic loading". J Geotech Geoenviron Eng, ASCE, 4014012-4014065

31. Hong Y, He B, Wang LZ, Wang Z, Ng CW, Masln D (2017) Cyclic lateral response and failure mechanisms of semi-rigid pile in soft clay: Centrifuge tests and numerical modelling. Can Geotech J 54(6):806-824

32. Hsu C-C, Vucetic M (2006) Threshold shear strain for cyclic pore-water pressure in cohesive soils. J Geotech Geoenviron Eng ASCE 132(10):1325-1335

33. Hsu C-C, Vucetic M (2004) Volumetric threshold shear strain for cyclic settlement. J Geotech Geoenviron Eng ASCE 132(1):58-70

34. Hyde AFL, Yasuhara K, Hirao K (1993) Stability criteria for marine clay under one-way cyclic loading. J Geotech Eng ASCE 119(11):1771-1789

35. Hyodo M, Hyde AFL, Yamamoto Y, Fujii T (1999) Cyclic shear strength of undisturbed and remoulded marine clays. Soils Found 39(2):45-58

36. Ichii K, Mikami T (2018) Cyclic threshold shear strain in pore water pressure generation in clay in situ samples. Soils Found 58(3):756-765

37. Ishihara K, Yamazaki F (1980) Cyclic simple shear tests on saturated sand in multi-directional loading. Soils Found 20(1):45-59

38. Jian-Hua Yin, Graham J (1994) Equivalent times and one-dimensional elastic viscoplastic modelling of time-dependent stress-strain behaviour of clays. Can Geotech J 31(1):42-52

39. Jostad HP, Dahl BM, Page A, Sivasithamparam N, Sturm H (2020) Evaluation of soil models for improved design of offshore 
wind turbine foundations in dense sand. Geotechnique 70(8):682-699

40. Lai Y, Wang L, Hong Y, He B (2020) Centrifuge modeling of the cyclic lateral behavior of large-diameter monopiles in soft clay: effects of episodic cycling and reconsolidation. Ocean Eng 200:107048

41. Liao W, Zhang J, Wu J, Yan K (2018) Response of flexible monopile in marine clay under cyclic lateral load. Ocean Eng 147:89-106

42. Lim G (2003) Stabilisation of an excavation by an embedded improved soil layer. Ph.D thesis. National University of Singapore, p. 219. https://scholarbank.nus.edu/handle/10635/13635

43. Machacek J, Staubach P (2021) Numgeo: a finite-element program for the simulation of hydro-mechanically coupled geotechnical processes. In: Fachsektionstage Geotechnik 2021. DGGT. https://www.numgeo.de/wp-content/uploads/2021/04/ 20210417_MachacekStaubach_FSTB2021.pdf

44. Machacek J, Wichtmann T, Zachert H, Triantafyllidis T (2018) Long-term settlements of a ship lock: measurements vs. FEprediction using a high cycle accumulation model. Comput Geotech 97(5):222-232

45. Machacek J, Staubach P, Tafili M, Zachert H, Wichtmann T (2021) Investigation of three sophisticated constitutive soil models: From numerical formulations to element tests and the analysis of vibratory pile driving tests. Comput Geotech 138:104276

46. Masin D (2013) Clay hypoplasticity with explicitly defined asymptotic states. Acta Geotech 8(5):481-496

47. Matlock H (1970) Correlations for design of laterally loaded piles in soft clay. In: Proceedings of the Annual Offshore Technology Conference. Vol. 1970-April. Offshore Technology Conference Inc., Houston, OTC-1204-MS. ISBN: 9781555637095

48. Matsuda H, ThanhNhan T, Ishikura R (2013) Prediction of excess pore water pressure and post-cyclic settlement on soft clay induced by uni-directional and multi-directional cyclic shears as a function of strain path parameters. Soil Dyn Earthq Eng 49:75-88

49. Matsui T, Ohara H, Ito T (1980) Cyclic stress-strain history and shear characteristics of clay. J Geotech Eng Div ASCE 106(GT10): 1101-1120

50. Medicus G, Fellin W (2017) An improved version of barodesy for clay. Acta Geotechnica 12(2):365-376. https://doi.org/10.1007/ s11440-016-0458-4

51. Mortezaie AR, Vucetic M (2013) Effect of frequency and vertical stress on cyclic degradation and pore water pressure in clay in the NGI simple shear device. J Geotech Geoenviron Eng ASCE 139(10): 1727-1737

52. Ng CW, Liu GB, Li Q (2013) Investigation of the long-term tunnel settlement mechanisms of the first metro line in Shanghai. Can Geotech J 50(6):674684

53. Niemunis A (2003) Extended hypoplastic models for soils. Habilitation, Publications of the Institute of Foundation Engineering and Soil Mechanics, Ruhr-University Bochum, Issue No. 34

54. Niemunis A, Grandas-Tavera CE, Prada-Sarmiento LF (2009) Anisotropic visco-hypoplasticity. Acta Geotech 4:293-314

55. Niemunis A, Wichtmann T, Triantafyllidis T (2005) A high-cycle accumulation model for sand. Comput Geotech 32(4):245-263

56. Niemunis A, Herle I (1997) Hypoplastic model for cohesionless soils with elastic strain range. Mech Cohesive Friction Mater 2(4):279-299

57. Page AM, Klinkvort RT, Bayton S, Zhang Y, Jostad HP (2021) A procedure for predicting the permanent rotation of monopiles in sand supporting offshore wind turbines. Mar Sruct 75:102813

58. Puech A, Garnier J (2017) Behavior of Piles Subject to Cyclic Lateral Loading. Design of Piles Under Cyclic Loading. Wiley. Chap. 8, pp 233-297
59. Puech A, Garnier J (2017) Design of Piles Subject to Cyclic Lateral Loading. In: Design of Piles Under Cyclic Loading. Wiley. Chap. 9, pp 299-346. ISBN: 9781119469018

60. Reese LC, Cox WR, Koop FD (1975) Field testing and analysis of laterally loaded piles in stiff clay. Tech. rep. paper 2312. Offshore Technology Centre, Dallas

61. Seidalinov G, Taiebat M (2014) Bounding surface SANICLAY plasticity model for cyclic clay behavior. Int J Numer Anal Methods Geomech 38:702-724

62. Staubach P, Machacek J (2019) Influence of relative acceleration in saturated sand: analytical approach and simulation of vibratory pile driving tests. Comput Geotech 112:173-184

63. Staubach P, Machacek J, Sharif R, Wichtmann T (2021) Backanalysis of model tests on piles in sand subjected to long-term lateral cyclic loading: impact of the pile installation and application of the HCA model. Comput Geotech 134:104018

64. Staubach P, Machacek J, Moscoso MC, Wichtmann T (2020) Impact of the installation on the long-term cyclic behaviour of piles in sand: a numerical study. Soil Dyn Earthq Eng 138:106223

65. Staubach P, Machacek J, Skowronek J, Wichtmann T (2021) Vibratory pile driving in water-saturated sand: back-analysis of model tests using a hydro-mechanically coupled CEL method. Soils Found 61(1):144-159

66. Staubach P, Machacek J, Tschirschky L, Wichtmann T (2021) Enhancement of a high-cycle accumulation model by an adaptive strain amplitude and its application to monopile foundations. Int $\mathbf{J}$ Numer Anal Methods Geomech. https://doi.org/10.1002/nag. 3301

67. Staubach P, Machacek J, Wichtmann T (2022) Novel approach to apply existing constitutive soil models to the modelling of interfaces. Int J Numer Anal Methods Geomech, under review

68. Staubach P, Wichtmann T (2020) Long-term deformations of monopile foundations for offshore wind turbines studied with a high-cycle accumulation model. Comput Geotech 124:103553

69. Staubach P, Machacek J, Wichtmann T (2021) Large-deformation analysis of pile installation with subsequent lateral loading: Sanisand vs. Hypoplasticity. Soil Dyn Earthq Eng 151:106964

70. Su D, Wu WL, Du ZY, Yan WM (2014) Cyclic degradation of a multidirectionally laterally loaded rigid single pile model in compacted clay. J Geotech Geoenviron Eng 140(5):06014002

71. M. Tafili (2020) On the behaviour of cohesive soils: Constitutive description and experimental observations. Dissertation, Publications of the Institute of Soil Mechanics and Rock Mechanics, Karlsruhe Institute of Technology, Issue No. 186

72. Tafili M, Fuentes W, Triantafyllidis T (2020) A comparative study of different model families for the constitutive simulation of viscous clays. Int $\mathrm{J}$ Numer Anal Methods Geomech 44:633-667

73. Tafili M, Triantafyllidis T (2020) AVISA: Anisotropic Visco ISA model and its performance at cyclic loading. Acta Geotech 15:2395-2413

74. Tafili M, Triantafyllidis T (2020) A simple hypoplastic model with loading surface accounting for viscous and fabric effects of clays. Int J Numer Anal Methods Geomech 44(16):2189-2215

75. Tafili M, Wichtmann T, Triantafyllidis T (2021) Experimental investigation and constitutive modeling of the behaviour of highly plastic lower Rhine clay under monotonic and cyclic loading. Can Geotech J 58(9):1396-1410. https://doi.org/10. 1139/cgj-2020-0012

76. Vucetic M (1994) Cyclic threshold shear strains in soils. J Geotech Eng ASCE 120(12):2208-2228

77. Wang D, Bienen B, Nazem M, Tian Y, Zheng J, Pucker T, Randolph MF (2015) Large deformation finite element analyses in geotechnical engineering. Comput Geotech-nics 65:104-114 
78. Wichtmann T (2016) Soil Behaviour under cyclic loading: experimental observations, constitutive description and applications. Habilitation thesis, Institute of Soil Mechanics and Rock Mechanics, Karlsruhe Institute of Technology, Issue No. 181

79. Wichtmann T, Machacek J, Zachert H, Günther H (2018) Validierung eines hochzyklischen Akkumulationsmodells anhand von Modellversuchen und Messungen an realen Bauwerken. Bautechnik 96(2):160-175

80. Wichtmann T, Triantafyllidis T (2018) Monotonic and cyclic tests on Kaolin - a database for the development, calibration and verification of constitutive models for cohesive soils with focus to cyclic loading. Acta Geotech 13(5):1103-1128

81. Wichtmann T, Niemunis A, Triantafyllidis T (2015) Improved simplified calibration procedure for a high-cycle accumulation model. Soil Dyn Earthq Eng 70(3):118-132

82. Wichtmann T, Steller K, Triantafyllidis T (2020) On the influence of the sample preparation method on strain accumulation in sand under high-cyclic loading. Soil Dyn Earthquake Eng 131(1):235-252

83. Wichtmann $\mathrm{T}$, Triantafyllidis $\mathrm{T}$, Chrisopoulos $\mathrm{S}$, Zachert $\mathrm{H}$ (2017) Prediction of long-term deformations of offshore wind power plant foundations using HCA-based engineer-oriented models. Int J Offshore Polar Eng 27(4):346356

84. Xiao J, Juang CH, Wei K, Xu S (2014) Effects of principal stress rotation on the cumulative deformation of normally consolidated soft clay under subway traffic loading. J Geotech Geoenviron Eng ASCE 140(4):4013046-4013049
85. Jie Yang Q, Feng Gao Y, GiongKong D, Zhu B (2019) Centrifuge modelling of lateral loading behaviour of a "semi-rigid" Monopile in soft clay". Mar Georesour Geotechnol, 37(10): 1205-1216

86. Yin J-H, Graham J (1999) Elastic viscoplastic modelling of the time-dependent stress-strain behaviour of soils. Can Geotech J 36(4):736-745

87. Zergoun M, Vaid YP (1994) Effective stress response of clay to undrained cyclic loading. Can Geotech J 31:714-727

88. Zhang Y, Andersen KH, Jeanjean P (2020) Verification of a framework for cyclic p-y curves in clay by hindcast of Sabine River, SOLCYP and centrifuge laterally loaded pile tests. Appl Ocean Res 97:102085

89. Zhang Y, Andersen KH, Jeanjean P, Karlsrud K, Haugen T (2020) Validation of monotonic and cyclic p-y framework by lateral pile load tests in stiff, overconsolidated clay at the haga site. J Geotech Geoenviron Eng 146(9):04020080

90. Zhou J, Gong X (2001) Strain degradation of saturated clay under cyclic loading. Can Geotech J 38:208-212

91. Zhu B, Zhu Z-J, Li T, Liu J-C, Liu Y-F (2017) Field tests of offshore driven piles subjected to lateral monotonic and cyclic loads in soft clay. J Waterway Port Coast Ocean Eng 143(5):05017003

Publisher's Note Springer Nature remains neutral with regard to jurisdictional claims in published maps and institutional affiliations. 